In Situ

Revue des patrimoines

\section{In Situ}

Revue des patrimoines

17 | 2011

Les patrimoines de l'enseignement supérieur

\title{
Fresques des salles de garde
}

Artistes et médecins, médecins artistes

\section{Christian Hottin}

\section{(2) OpenEdition \\ Journals}

Édition électronique

URL : http://journals.openedition.org/insitu/955

DOI : 10.4000/insitu. 955

ISSN : 1630-7305

Éditeur

Ministère de la culture

Référence électronique

Christian Hottin, «Fresques des salles de garde », In Situ [En ligne], 17 | 2011, mis en ligne le 22 juin 2016, consulté le 20 avril 2019. URL : http://journals.openedition.org/insitu/955 ; DOI : 10.4000/ insitu.955

Ce document a été généré automatiquement le 20 avril 2019

\section{(c) $($ ) $(9)$}

In Situ Revues des patrimoines est mis à disposition selon les termes de la licence Creative Commons Attribution - Pas d'Utilisation Commerciale - Pas de Modification 4.0 International. 


\title{
Fresques des salles de garde
}

\author{
Artistes et médecins, médecins artistes
}

\section{Christian Hottin}

1 La compréhension des œuvres d'art peintes ou sculptées exécutées par des artistes professionnels pour les membres d'un groupe humain constitué en institution passe par l'analyse des rapports multiformes de cette communauté avec l'art. Rapport pratique : les membres de la communauté ne disposent pas eux-mêmes, sauf exceptions collectives ou individuelles, de la capacité technique leur permettant de produire l'œuvre d'art en autarcie. Sa réalisation passe alors par un processus de commande directe ou indirecte, selon qu'elle réside dans la simple mise en rapport des instances dirigeantes du groupe avec l'artiste, ou fait intervenir une autre institution qui assure la médiation en direction des praticiens, attribue les commandes, finance les travaux et exerce une action de contrôle sur la réalisation des œuvres ${ }^{1}$. Rapport symbolique : l'œuvre d'art, en raison (entre autres) de sa visibilité, de son coût et du prestige attaché au travail artistique, peut constituer un support pérenne des représentations élaborées par le groupe à l'intention de ses membres ou en direction de l'extérieur (autres communautés ou collectivités plus vastes telles que la Nation). Créée pour participer à la constitution de l'identité collective (fig. $\left.n^{\circ} 1\right)\left(\right.$ fig. $n^{\circ} 2$ ), elle peut devenir, par le biais d'une réappropriation patrimoniale, élément constitutif du sentiment communautaire. 
Figure 1

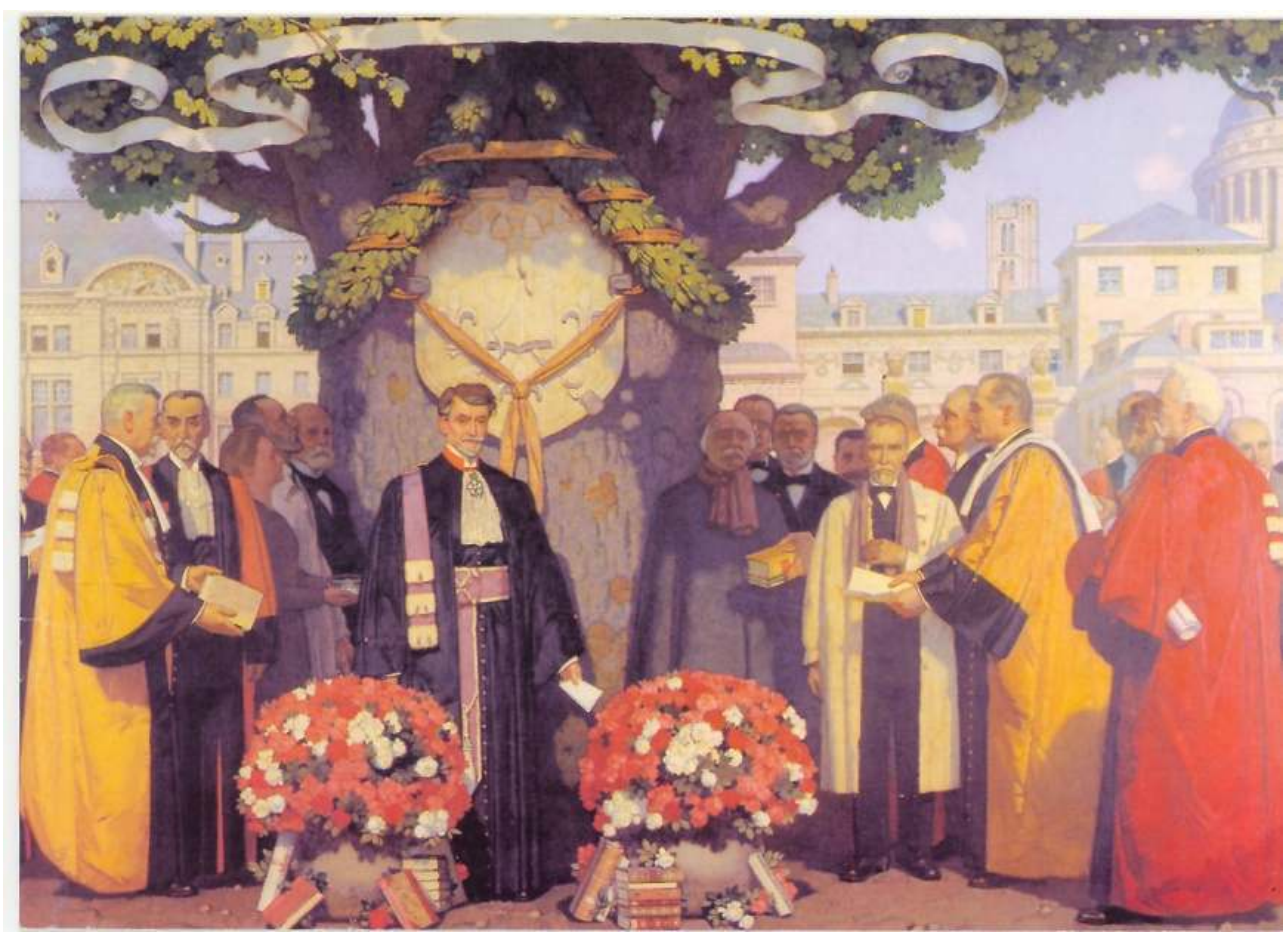

Un exemple de portrait collectif de la communauté universitaire : Georges Leroux, L'Université de Paris. Paris, Institut de Chimie [ENCP], 1936.

Phot. Jean-Christophe Doërr, 1999.

\section{Figure 2}

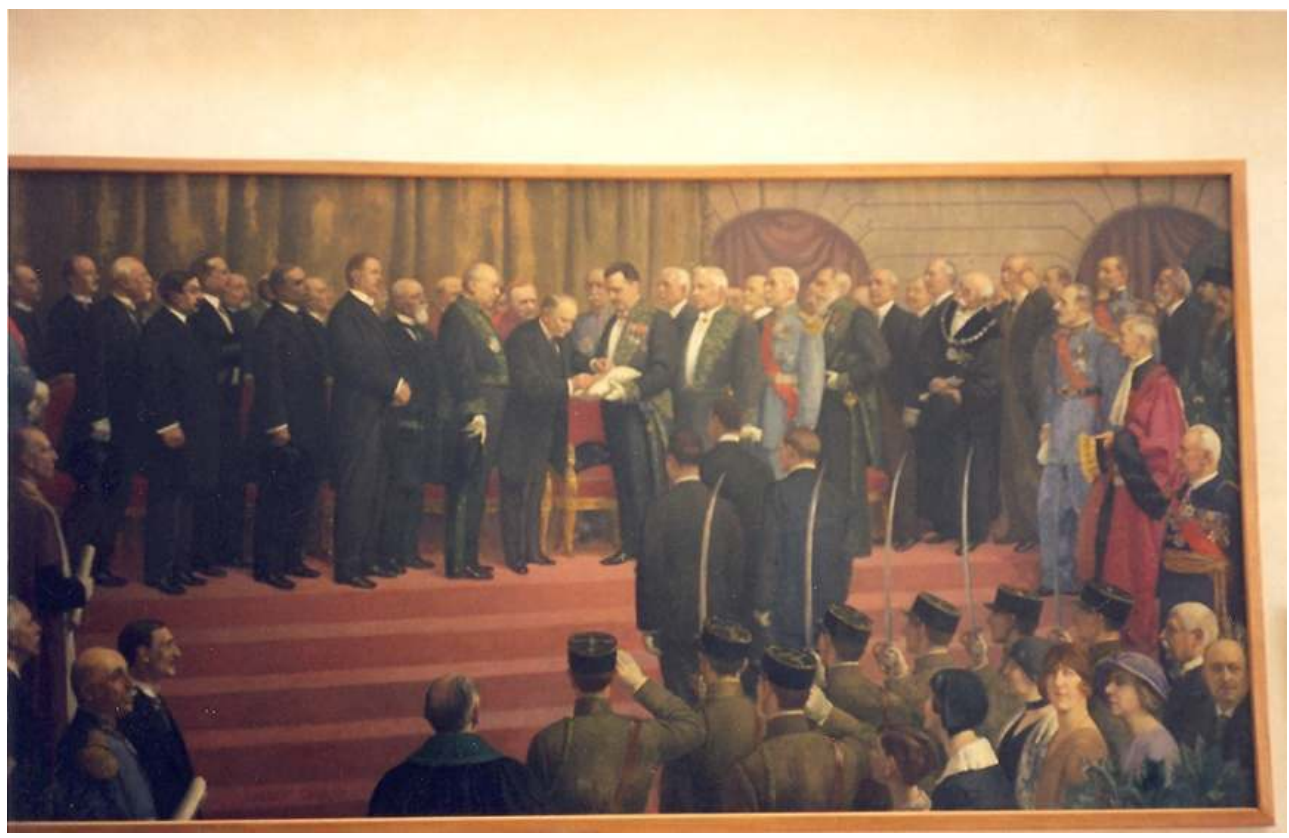

Un exemple de portrait collectif dans le monde des grandes écoles : Paul-Albert Laurens, La remise de la croix de la Légion d'Honneur à l'École centrale des Arts et manufactures. Châtenay-Malabry, École centrale des Arts et Manufactures.

Phot. Christian Hottin, 1995. 
Deux intuitions, développées en hypothèses de travail et étayées lors d'une recherche antérieure ${ }^{2}$ portant sur les décors des palais universitaires, supposaient, d'une part, que les représentations supportées par les œuvres d'art ne pouvaient être dissociées de celles développées via d'autres médias utilisés par les groupes humains étudiés (principalement les textes et les cérémonies assimilables à des rites collectifs) et, d'autre part, que ces représentations, quel que soit leur mode d'expression, s'articulaient autour des mêmes thématiques, quelle que soit la position relative de leurs producteurs au sein du groupe (élèves, anciens élèves, professeurs, dirigeants administratifs). (fig. $\left.\mathbf{n}^{\circ} \mathbf{3}\right)$ (fig. $\left.\mathbf{n}^{\circ} \mathbf{4}\right)$ De là est né un intérêt plus particulier pour les artefacts produits par ou pour les élèves des écoles supérieures ${ }^{3}$, dans le but de les confronter aux représentations élaborées par les dirigeants des institutions et de rechercher des points de comparaison en dehors du cadre primitif de l'étude, limité alors aux établissements d'enseignement supérieur.

Figure 3

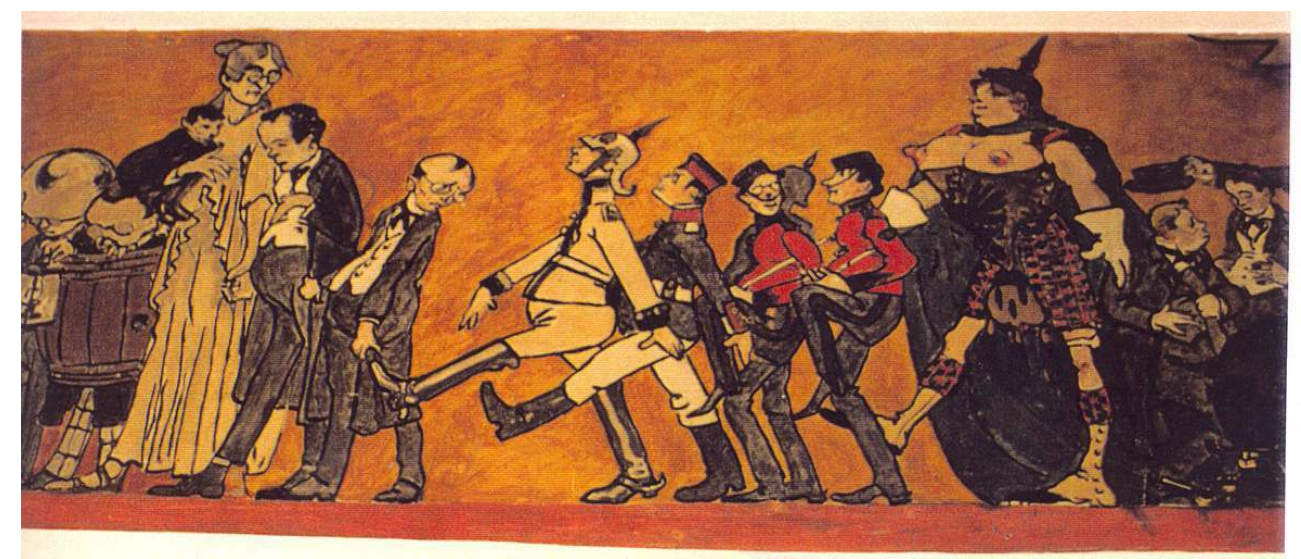

Un exemple de portrait collectif réalisé avec le concours des élèves : Pierre-Victor Robiquet, AndréFrançois Poncet et al., [Philosophes et germanistes de Normale sup'se rendant à l'Agrégation]. Paris, École normale supérieure.

Phot. Philippe Reix, 1995. 


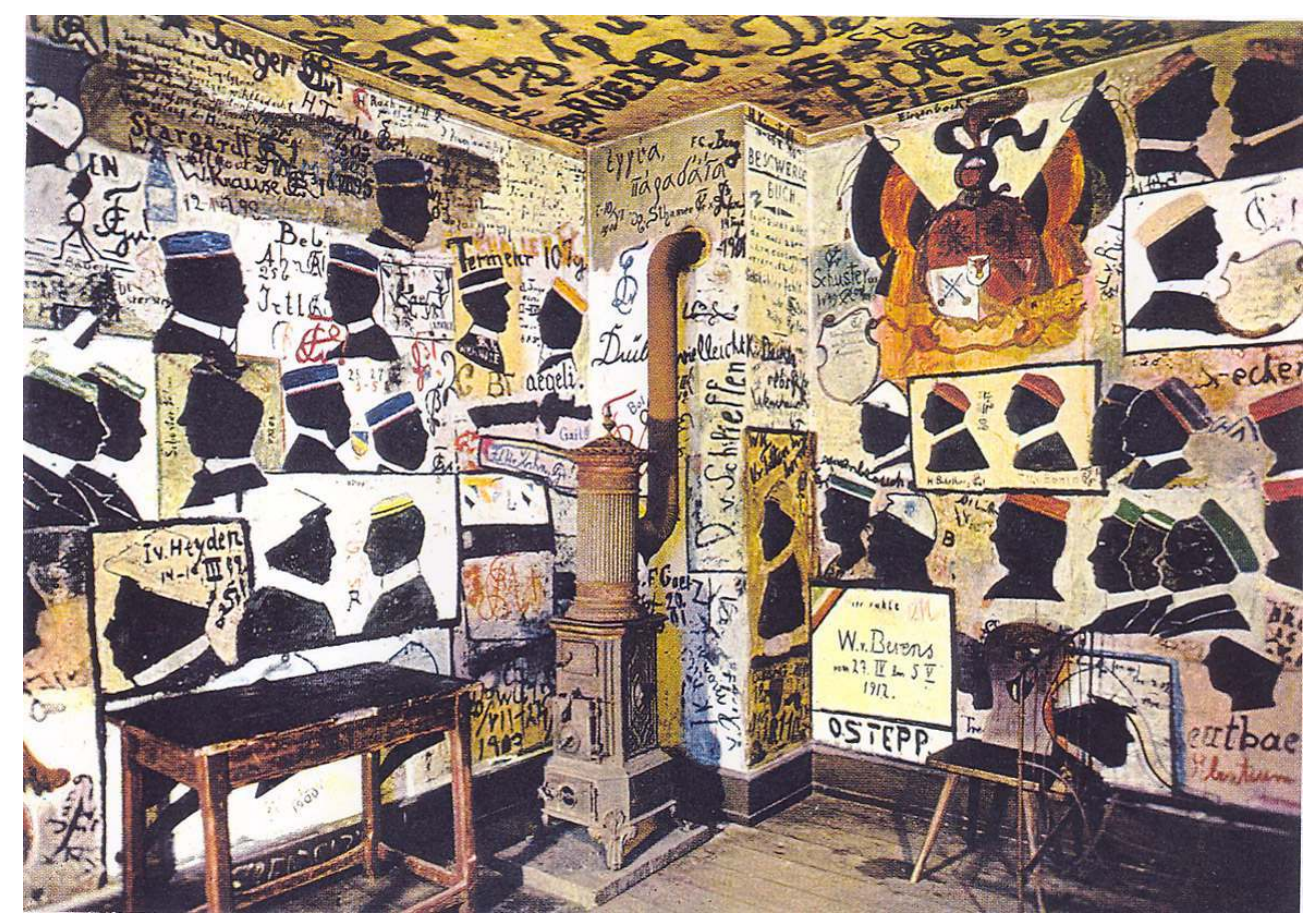

Un exemple de collection de portraits réalisée par les étudiants : an., Le cachot des étudiants de I'Université de Heidelberg. Heidelberg, République fédérale d'Allemagne.

Phot. Christian Hottin, 1982

3 La découverte des fresques ${ }^{4}$ des salles de garde des hôpitaux parisiens a ouvert un champ de recherche susceptible de recevoir un traitement autonome, tant le phénomène est différent du corpus initial par les modes de production, les thématiques développées, leurs modalités d'expression ou encore les possibilités d'interprétations sociologiques ou ethnologiques attachées aux œuvres. (fig. $\mathbf{n}^{\circ} 5$ ) En premier lieu, ce phénomène, en raison de son inscription dans la durée et de sa vitalité toujours actuelle, permet d'observer les processus de commande et d'exécution des peintures in situ et de les confronter dans une approche diachronique avec d'autres types de sources de la recherche historique. En outre, les fresques s'insèrent dans les rites complexes de la salle de garde, qui est ellemême un des traits constitutifs du groupe des internes et anciens internes, lesquels occupent une position essentielle dans la hiérarchie des études de médecine et dans l'organisation de la pratique hospitalière. Tout en étant le résultat d'un processus de création complexe, il s'inscrit dans un contexte sociologique riche, ce qui lui confère une résonance particulière. En somme, sans prétendre le moins du monde voir dans la fresque une clef d'analyse du champ social que constitue le monde médical, son interprétation passe par la prise en compte de ces différents éléments. Enfin, l'analyse et l'interprétation des fresques est rendue plus délicate par l'existence de nombreux autres supports de représentation produits par les étudiants dans le cadre de leurs années d'internat. Les chansons ${ }^{5}$ et les rites ${ }^{6}$ sont les plus connus. Ces derniers sont également l'occasion de produire des représentations éphémères (chars construits pour les bals annuels) ou durables (cartes d'invitations aux bals) ${ }^{7}$. La très abondante littérature historique ou mémorielle élaborée par les " fossiles $»^{8}$ à l'attention de leurs pareils occupe également une place privilégiée parmi les sources comparatives à explorer : souvenirs de patrons et histoires de l'Internat sont autant de discours historiques linéaires, dépourvus de toute 
recherche d'objectivation de l'expérience, mais qui incluent dans leurs développements des éléments caractéristiques de la définition identitaire du groupe, tout en leur donnant une nouvelle forme. Cet énième avatar constitue une nouvelle formulation littéraire des représentations (en l'occurrence un type de discours sur la salle de garde qui se veut historique), mais c'est aussi la mise en forme d'un nouvel objet tridimensionnel, susceptible de faire l'objet d'une approche codicologique (par exemple des ouvrages luxueusement reliés, dorés aux fers et imprimés sur papier chiffon, et présentés dans un coffret recouvert de velours grenat). Ces livres apparaissent donc comme une source d'informations plus ou moins utiles pour la connaissance de l'histoire du phénomène, mais ils sont également une série d'artefacts supplémentaire à prendre en compte dans l'analyse ${ }^{9}$.

Figure 5

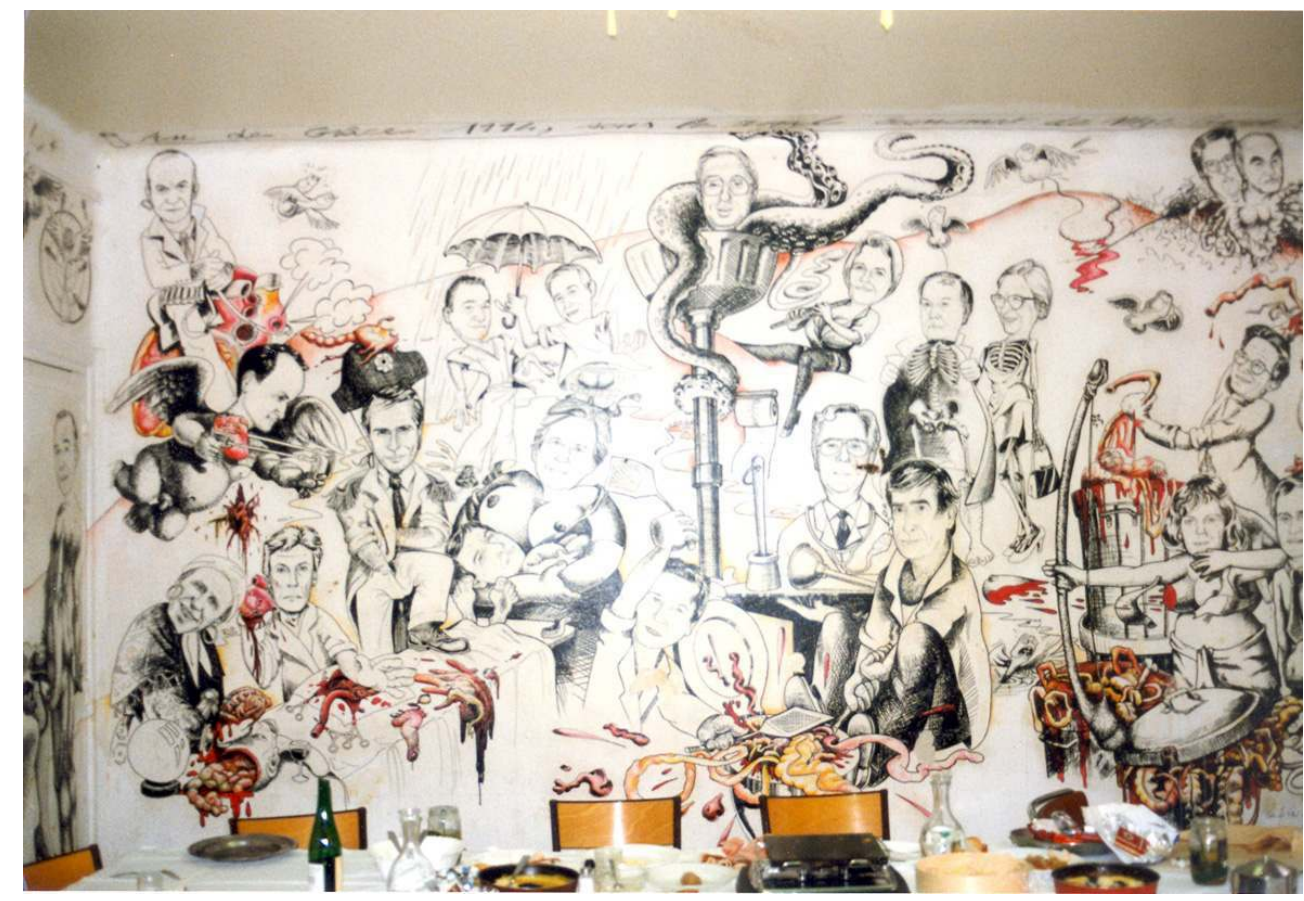

Christine Boulanger. Portrait collectif. Paris, salle de garde de l'Hôpital Broussais.

Phot. Christian Hottin, 1998.

4 Leur inscription dans la longue durée historique, leur participation à des rapports de forces qui structurent en profondeur le champ du monde médical et la présence de nombreux supports de représentation susceptibles d'être mis en rapport avec elles, sont autant d'éléments à prendre en compte pour livrer une interprétation des fresques des salles de garde. Toutefois, ce qu'elles sont susceptibles de livrer n'est pas une explication globale des mécanismes qui sous-tendent la vie des internes en médecine, mais plutôt un éclairage sur les rapports que ce groupe (et, dans une certaine mesure, le monde médical dans son ensemble) entretient avec différentes notions telles que le pouvoir, l'art ou la sexualité.

5 Une double médiation artistique, c'est-à-dire le recours à un artiste professionnel pour exécuter la peinture et l'expression de l'identité communautaire à travers l'œuvre d'art, est au cœur du processus de production des fresques : c'est par ce rapport à l'art et à l'artiste que débute notre parcours. Comprendre cette relation suppose toutefois de 
savoir en quoi consiste une fresque et comment se déroule une commande de décor mural.

\section{Une salle de garde}

(fig. $\mathbf{n}^{\circ} \mathbf{6}$ ) La salle de garde est l'espace, au sein de l'hôpital, où les internes prennent leurs repas et leurs heures de repos pendant le temps des gardes ${ }^{10}$. Selon la date de construction de l'établissement ou la configuration des services, elle peut consister en un édifice indépendant (c'est le cas à Saint-Louis ou Saint-Antoine), occuper tout ou partie d'un pavillon (comme à l'ancien hôpital Boucicaut, où la salle donnait sur la rue de la Convention) ou encore une zone limitée dans un grand bâtiment (à l'Hôtel Dieu de Paris, la salle occupe une partie d'une aile donnant sur le parvis de Notre-Dame). Par métonymie, on nomme couramment «salle de garde" l'ensemble des locaux qui constituent en fait l'internat: outre la salle proprement dite, celui-ci comprend les cuisines, quelques chambres et, fréquemment, une ou deux pièces voisines de la salle et faisant office d'espace de détente (baby-foot, fauteuils). Depuis la création de l'Internat en 1802, la place de la salle de garde dans l'existence des internes à évolué à mesure que se modifiaient certains aspects de leur profil sociologique: de lieu de vie permanent pour des jeunes célibataires de sexe masculin ${ }^{11}$, elle est devenue un point de passage quotidien pour des jeunes hommes et femmes souvent mariés et résidant hors de l'hôpital ${ }^{12}$.

\section{Figure 6}

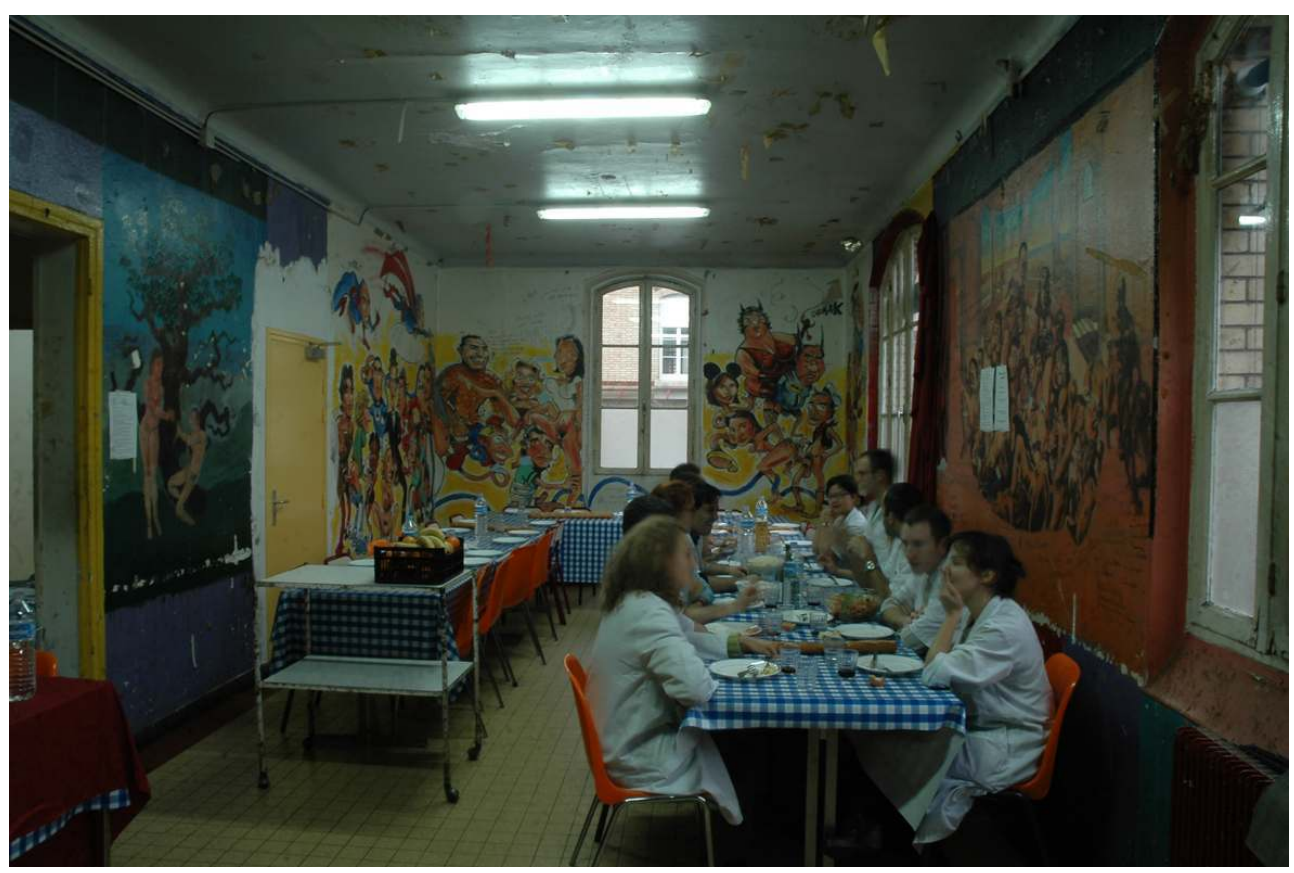

Un repas en salle de garde. Paris, Hôpital de la Pitié Salpétrière.

Phot. an. 2006 


\section{Figure 7}

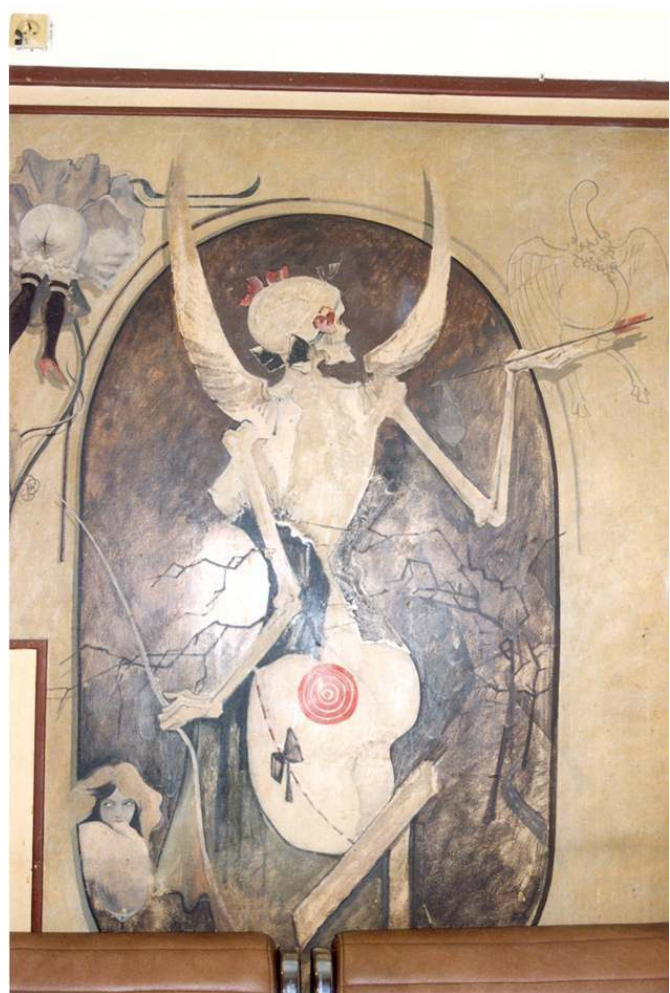

L'initiation sentimentale (d'après Rops). Paris, Hôpital Bichat. Phot. Christian Hottin, 1998.

7 Si les fresques constituent l'élément visuel le plus frappant d'une salle, elles ne sont pas le seul et entrent en relation avec d'autres objets ${ }^{13}$ et dispositifs rituels également employés par les internes lors de leur passage en salle. 
Figure 8

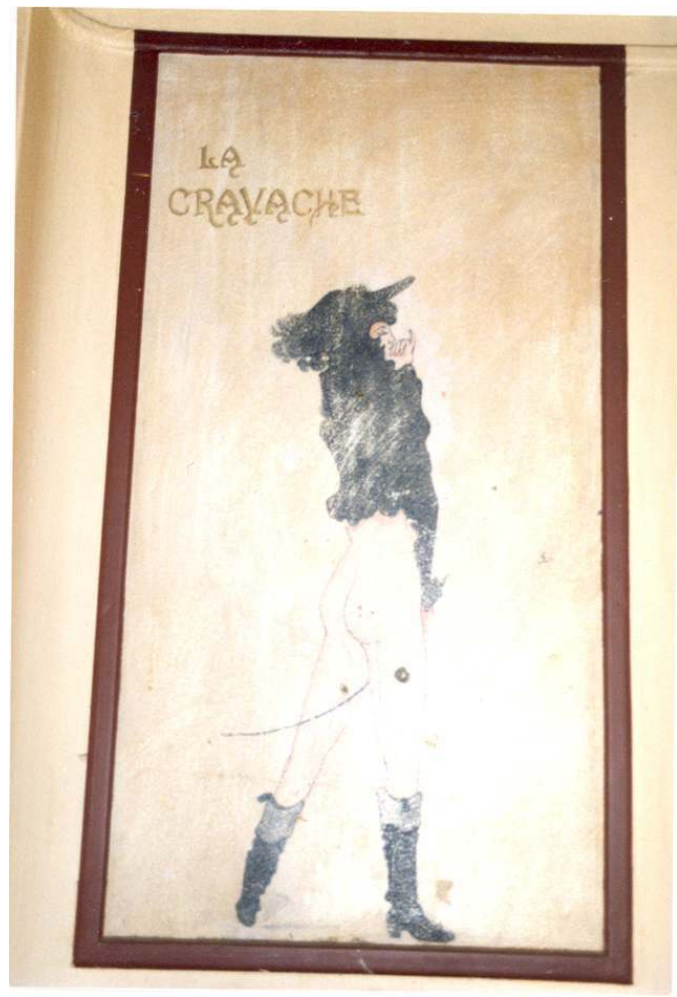

La Cravache. Paris, Hôpital Bichat.

Phot. Christian Hottin, 1998. 


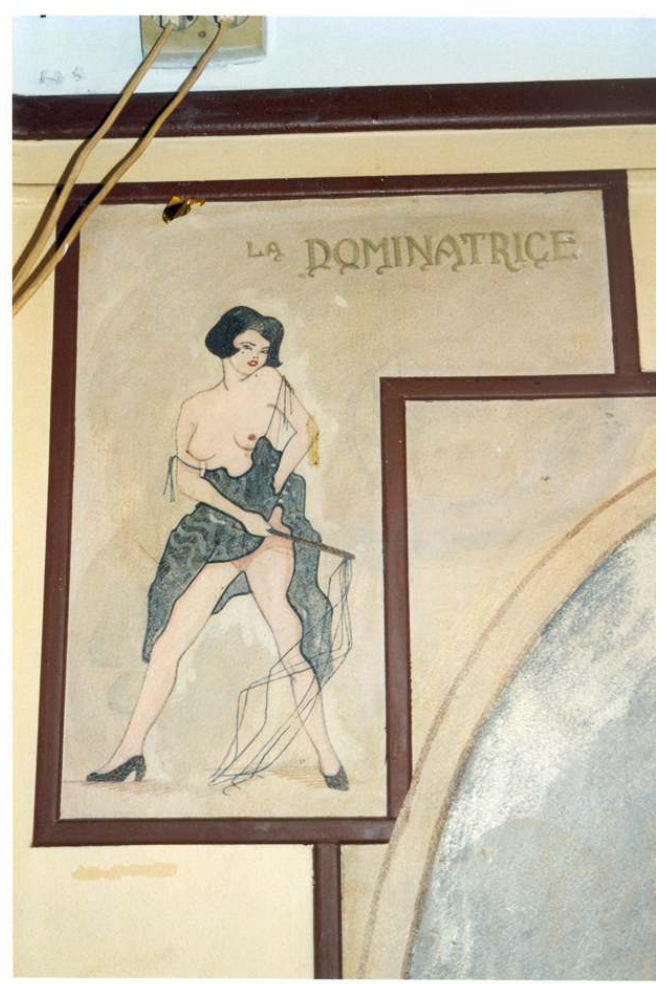

La Dominatrice. Paris, Hôpital Bichat.

Phot. Christian Hottin, 1998.

L'hôpital universitaire Bichat-Claude Bernard est situé au nord de Paris sur les boulevards des Maréchaux. Dans le bâtiment qu'elle occupe, on accède à la salle par un escalier proche des cuisines. La première pièce que l'on découvre est la salle à manger, de forme allongée. Sur son côté ouest, deux portes donnent accès à un salon équipé d'un bar et un bureau. Les chambres se trouvent au nord de cet ensemble. Dans le bar on découvre les plus anciennes peintures du site, signées « Germain 1964 ». Elles sont exécutées dans un style et représentent des sujets qui évoquent la fin du XIXe siècle. Les citations d'œuvres de peintres célèbres (fig. $\mathbf{n}^{\circ} \mathbf{7}$ ) (Klimt ou Rops, dont on voit une copie de L'initiation sentimentale ${ }^{14}$ ) voisinent avec des scènes légères. L'une d'elles montre une scène de bal, une autre l'intérieur d'une maison close (un homme avec une prostituée au salon). Elle est surmontée par une figure d'homme arborant de grands bois de cerf entre lesquels se balance une femme entièrement nue. Sur le même mur, trois autres, partiellement dévêtues et de plus petites dimensions, sont intitulées «La dominatrice» (tenant un fouet) (fig. $\mathbf{n}^{\circ} \mathbf{8}$ ), «La cravache " (en raison de son attribut) (fig. $\mathbf{n}^{\circ}{ }^{\circ}$ ) (fig. $\mathbf{n}^{\circ}{ }^{10}$ ) «L'espoir » (portant gaine et bas, elle est assise sur une chaise tendue de rouge, jambes écartées) $)^{15}$. 
Figure 10

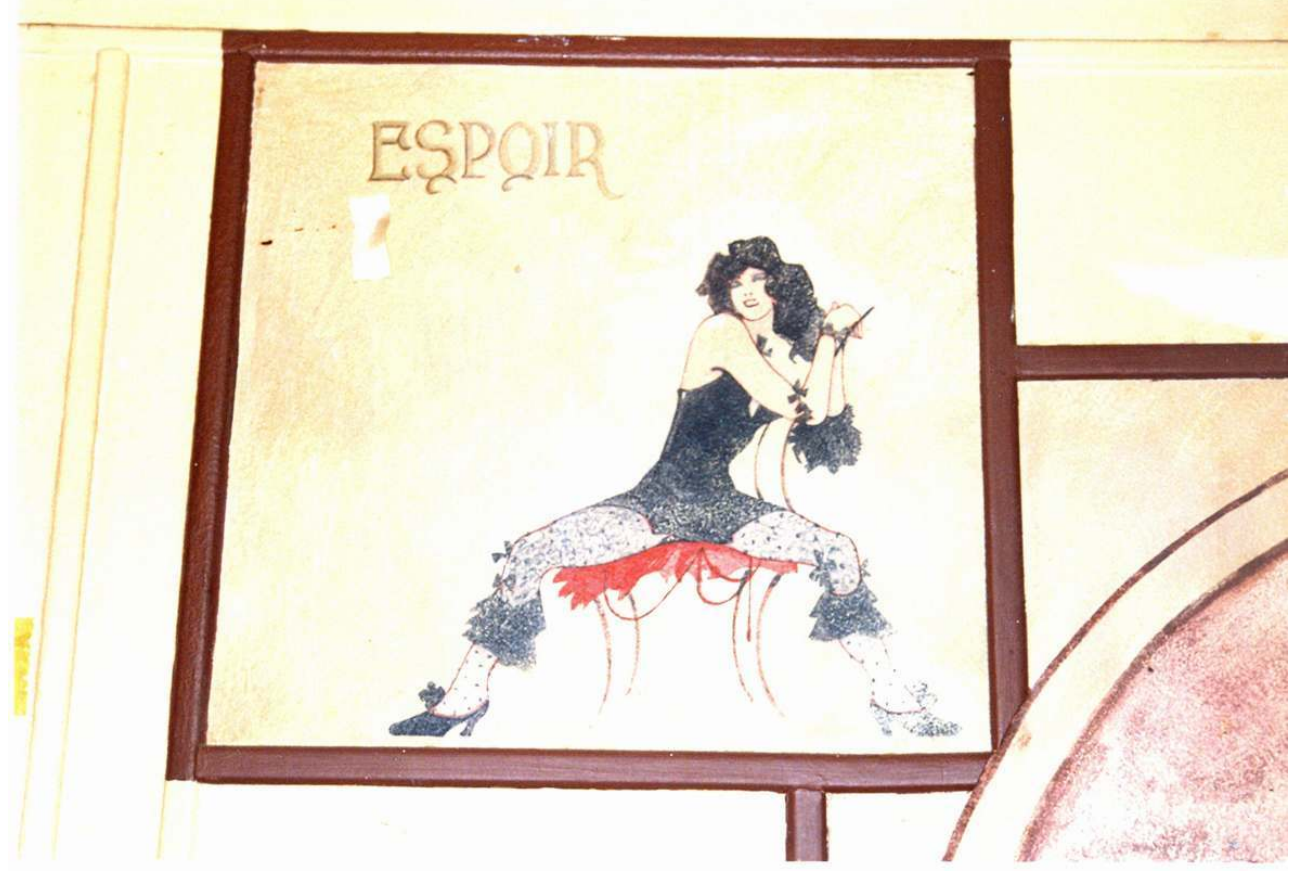

L'espoir. Paris, Hôpital Bichat.

Phot. Christian Hottin, 1998.

Figure 11

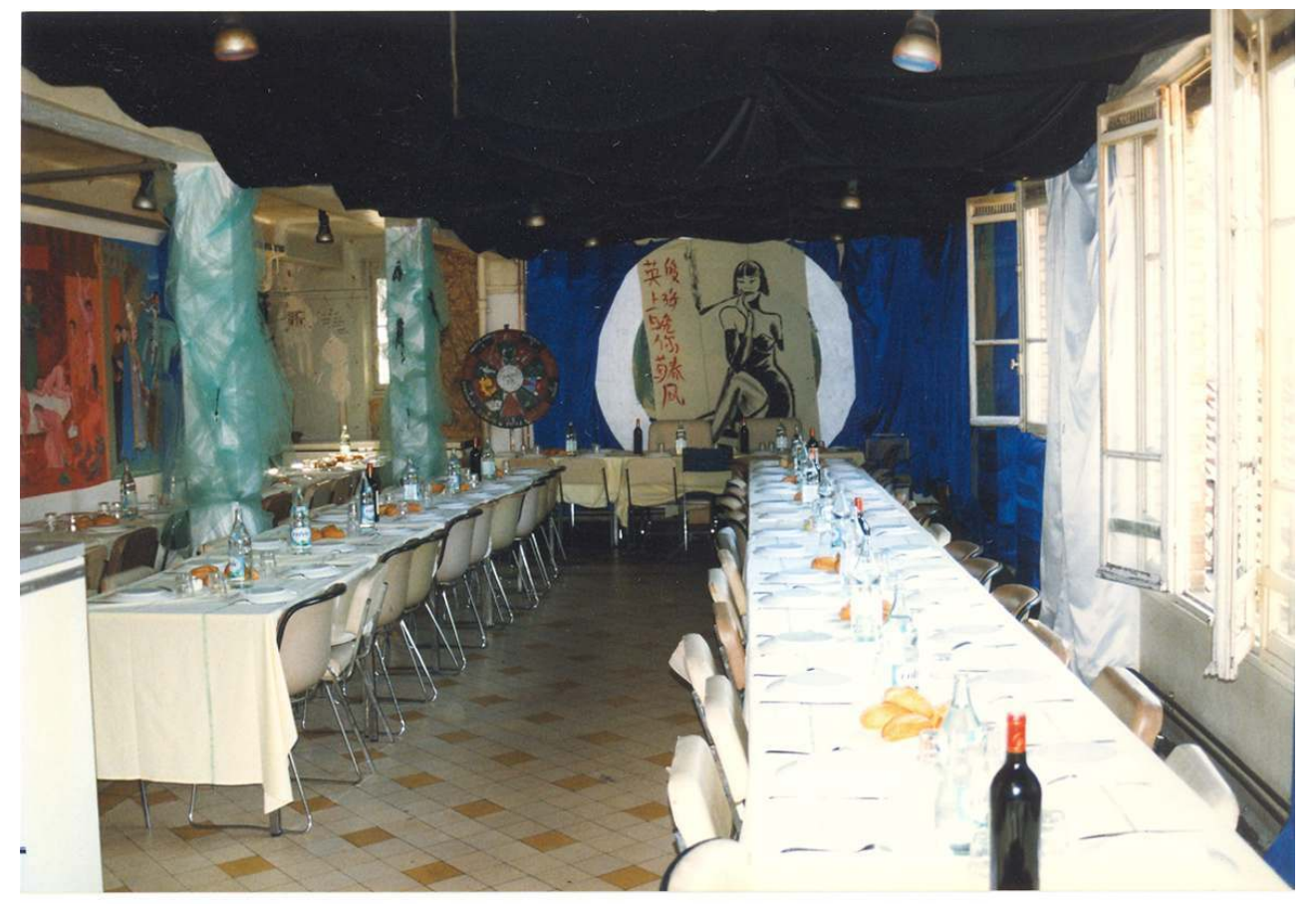

Une salle de garde préparée pour un tonus. Paris, Hôpital Bichat.

Phot. Christian Hottin, 1998. 
La salle principale, lieu des repas, offre des peintures beaucoup plus récentes. Lors de la visite des lieux ${ }^{16}$, elle est en grande partie tendue de satin bleu, en raison de la préparation d'un tonus ${ }^{17}$. Certaines fresques ne sont donc pas visibles (fig. $\left.\mathbf{n}^{\mathbf{0}} \mathbf{1 1}\right)$. Selon l'usage, toutes les tables sont jointes pour former un grand U sur trois côtés de la pièce. La table du fond est celle qu'occupe traditionnellement l'Économe ${ }^{18}$, désigné par les autres internes pour le semestre. À proximité de sa chaise est fixée sur le mur la roue ${ }^{19}$. (fig. $\mathbf{n}^{\circ}$ 12) Elle porte les taxes suivantes: "Pelle/Banco/Chansons/Histoire drôle/Roulette russe/Strip poker (cul ou poumon)/gâterie/mime sexuel/Passe /Vox populi ». Les différentes fresques occupent des espaces bien circonscrits, limités à une partie de chaque pan de mur. À droite de l'entrée apparaît la dernière fresque réalisée, une scène nautique dont le thème explique les tentures bleues de la pièce. La composition et l'exécution sont simples et presque naïves. Sur une mer calme apparaît une galère romaine. À la proue un personnage revêtu d'une cuirasse et tenant l'Aigle de Rome paraît imposer la cadence, relayé par un autre, à la poupe, qui bat la mesure sur un gros tambour. Au milieu du bateau se déroule une scène d'amour à trois, au rythme du tambour. Tout autour du navire, des femmes et des hommes s'accouplent à divers objets et créatures (poulpes, sirènes ou ordinateur), sous l'œil goguenard de morses eux-mêmes fort occupés. En général, les corps ne sont que grossièrement peints, tandis que les visages font l'objet de beaucoup d'attention, de manière à constituer des portraits ressemblants.

Figure 12

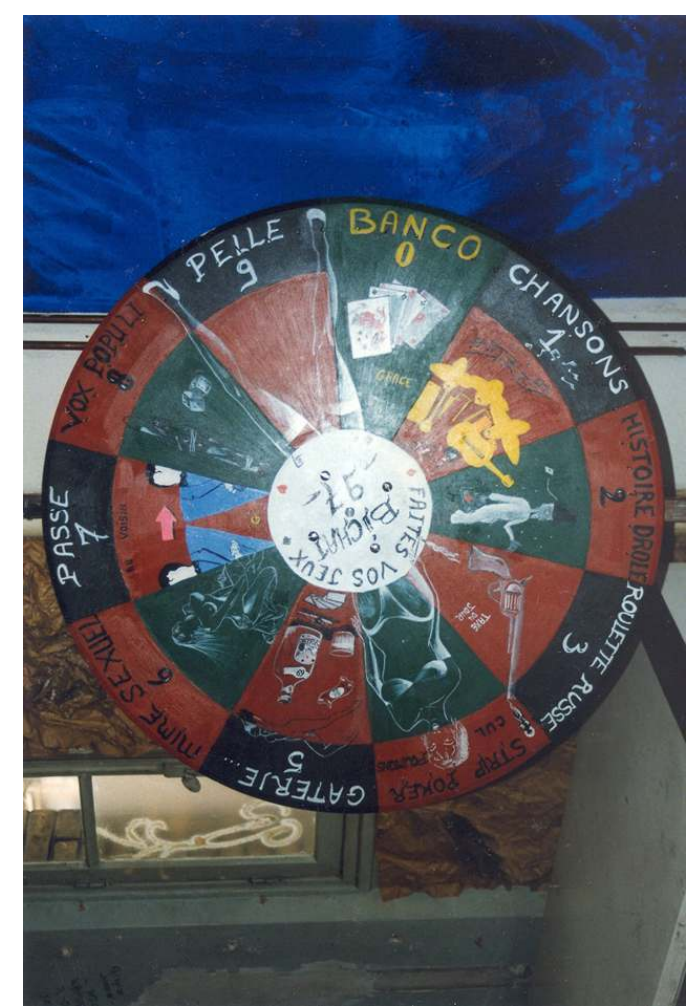

Une roue de salle de garde. Paris, Hôpital Bichat.

Phot. Christian Hottin, 1998. 
Figure 13

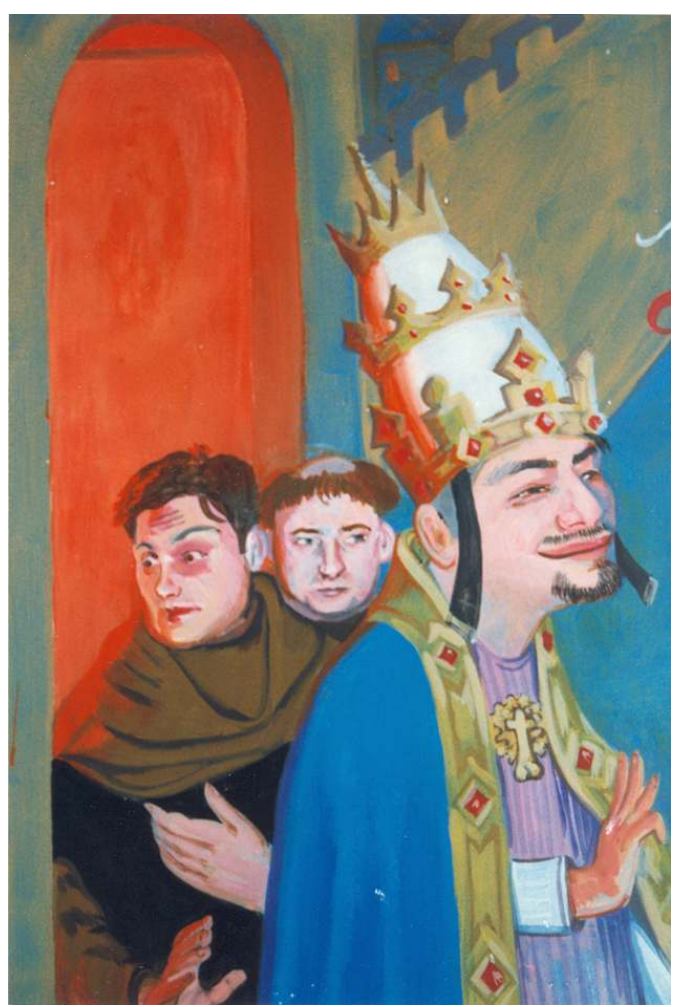

Portrait collectif médiéval, détail. Paris, Hôpital Bichat. Phot. Christian Hottin, 1998.

\section{Figure 14}

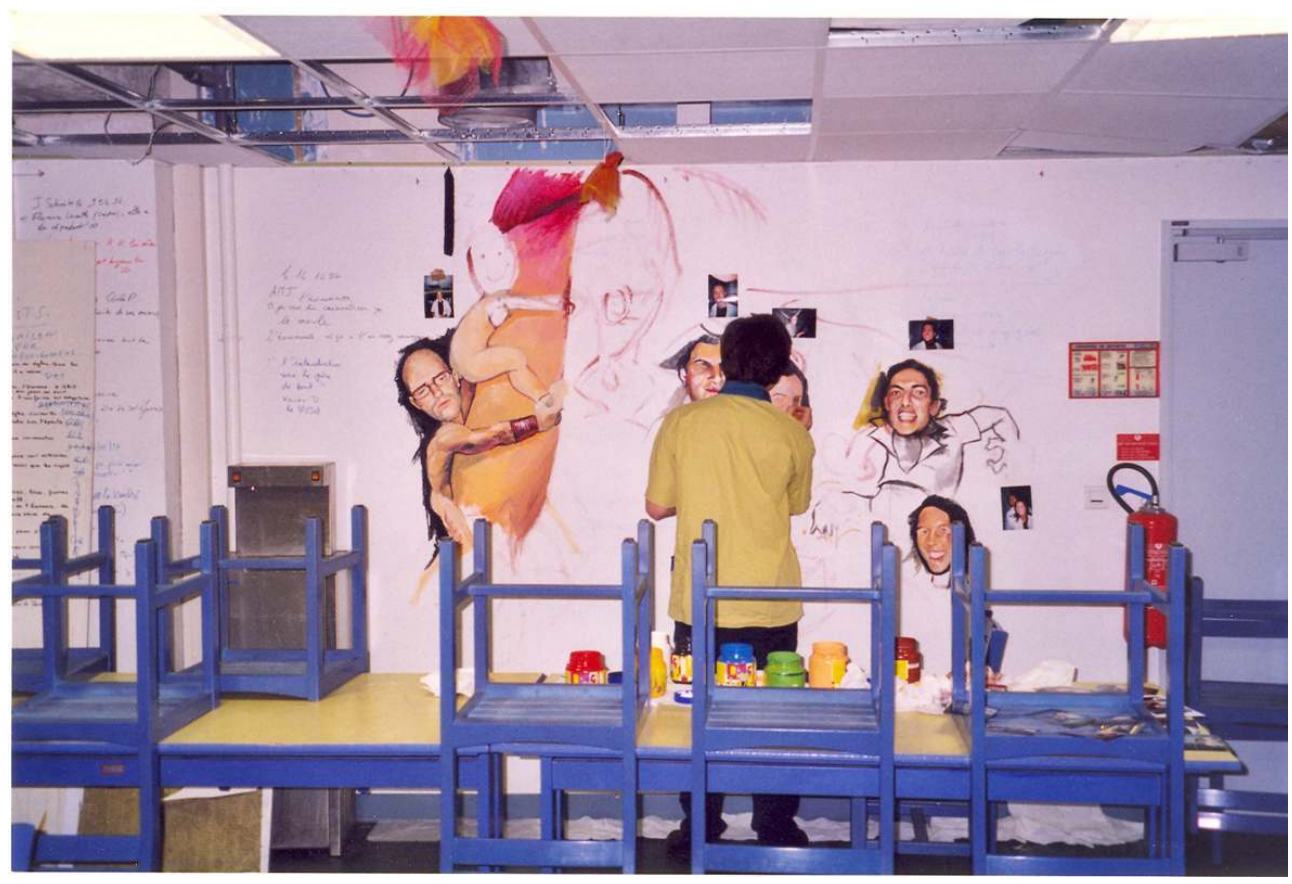

Grégory Maklès à l'œuvre pour décorer la salle de garde de l'Hôpital Necker. Paris, Hôpital Necker. Phot. Christian Hottin, 2000. 
Deux peintures occupent le mur situé à gauche de l'entrée. La première est datée et signée : «Cette fresque Avant la croisade festoy réalisey par le sieur Albrecht Wölf l'an de grâce mil-neuf-cent-quatre-vingt-dix-sept, octobre ». Dans une chambre rouge ayant un coffre et un lit à baldaquin rouge pour seul mobilier cinq couples font l'amour dans différentes positions. Les cinq femmes ont leur hennin pour tout vêtement, alors qu'une autre, encore habillée, s'apprête à rejoindre un homme couché sur le sol, torse nu. Un autre homme, debout sur le lit, présente sur un plat quatre ceintures de chasteté, tandis qu'un dernier, placé hors de la chambre, observe toute la scène par une fenêtre située à gauche. Deux moines offusqués se tiennent à droite dans l'embrasure de la porte, près de laquelle un pape "bénit» des chevaliers pour la croisade. (fig. n' ${ }^{\circ}$ ) Le style hésite entre le pastiche (avec une composition d'ensemble et un refus des lois de la perspective qui se veulent inspirés de l'art de la fresque médiévale) et la caricature (avec l'utilisation comique des coiffures et la reprise de quelques topoi des représentations contemporaines de la sexualité au Moyen Âge, telles que les clercs pudibonds et les ceintures de chasteté). La dernière peinture, plus petite, est placée tout près de la porte qui mène au bureau. Il s'agit d'un portrait de l'économinette ${ }^{20}:$ une jeune femme assise, la jupe relevée, un sein offert aux regards. Une main masculine lui enfonce une carotte dans le sexe.

Figure 15

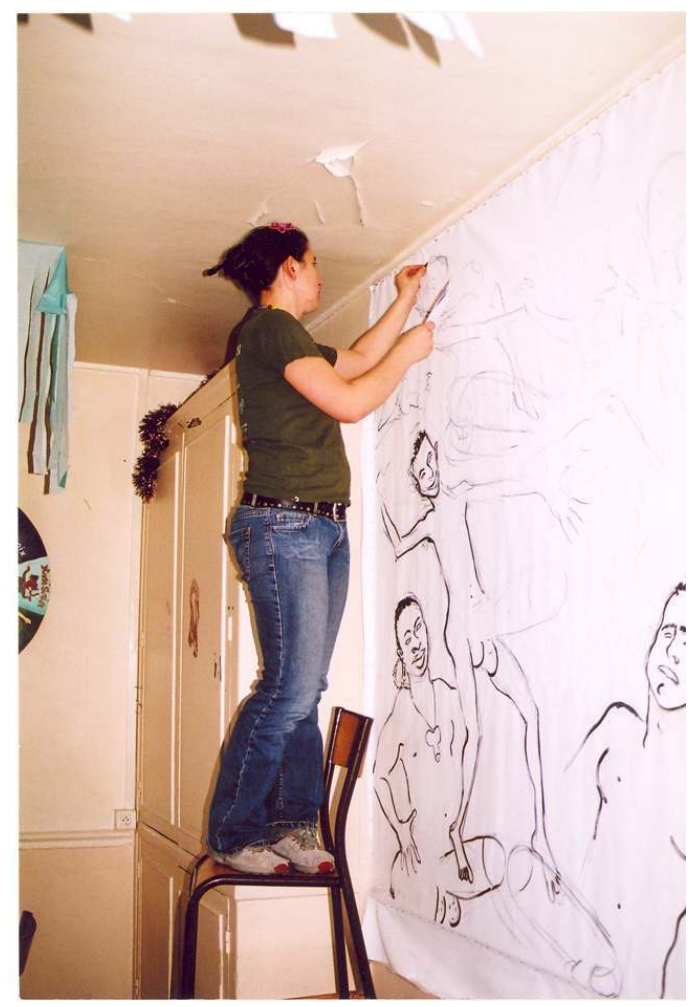

Une artiste à l'œuvre dans la salle de garde de l'Hôpital Lariboisière. Paris, Hôpital Lariboisière. Phot. Jean-Christophe Doërr, 2006.

\section{La commande}

11 Le processus de commande, d'exécution et (éventuellement) de destruction de la fresque est intimement lié au rythme des semestres passés par un groupe d'internes dans la salle 
de garde. Le groupe qui se trouve réuni pour six mois dans un hôpital désigne un économe qui sera chargé de recueillir les contributions des membres de la salle destinées à son approvisionnement (en particulier pour les repas hebdomadaires dénommés " améliorés »). Le tonus, moment essentiel du semestre, est le plus souvent organisé vers la fin de la période. À l'occasion de cette fête nocturne, l'économe peut commander à un artiste l'exécution d'une fresque, mais rien ne l'y oblige. Ainsi, à l'hôpital Armand Trousseau, trois nouvelles fresques seulement ont été exécutées entre 1988 et $1998^{21}$.

Le lien entre la fresque et le tonus est toutefois important, puisque le thème de l'un, choisi par l'économe, conditionne celui de l'autre ${ }^{22}$. L'économe entre en contact avec un peintre, souvent un élève de l'École des Beaux-Arts, et lui propose d'exécuter une fresque pour sa salle de garde ${ }^{23}$. Le prix varie en fonction de la taille de la fresque, il se situe en général entre 2000 et 5000 francs $^{24}$. En outre, l'artiste est nourri par la salle de garde pendant la durée de son travail. L'économe indique au peintre les contraintes liées à son projet $^{25}$, soit le respect du thème imposé, et la quasi obligation de traiter le thème à travers le prisme de l'activité sexuelle tout en faisant figurer sur la fresque les internes qui ont vécu dans la salle au cours du semestre écoulé. La fresque est donc un portrait de groupe, qui peut représenter d'emblée une situation liée à l'acte sexuel (c'est le cas de l'orgie de Boucicaut), mais peut également apparaître comme le détournement d'une situation historique (on l'a vu à Bichat avec la galère romaine et le départ pour la croisade). Enfin, est rappelée au peintre la nécessité de faire des portraits très ressemblants des internes ${ }^{26}$.

Figure 16

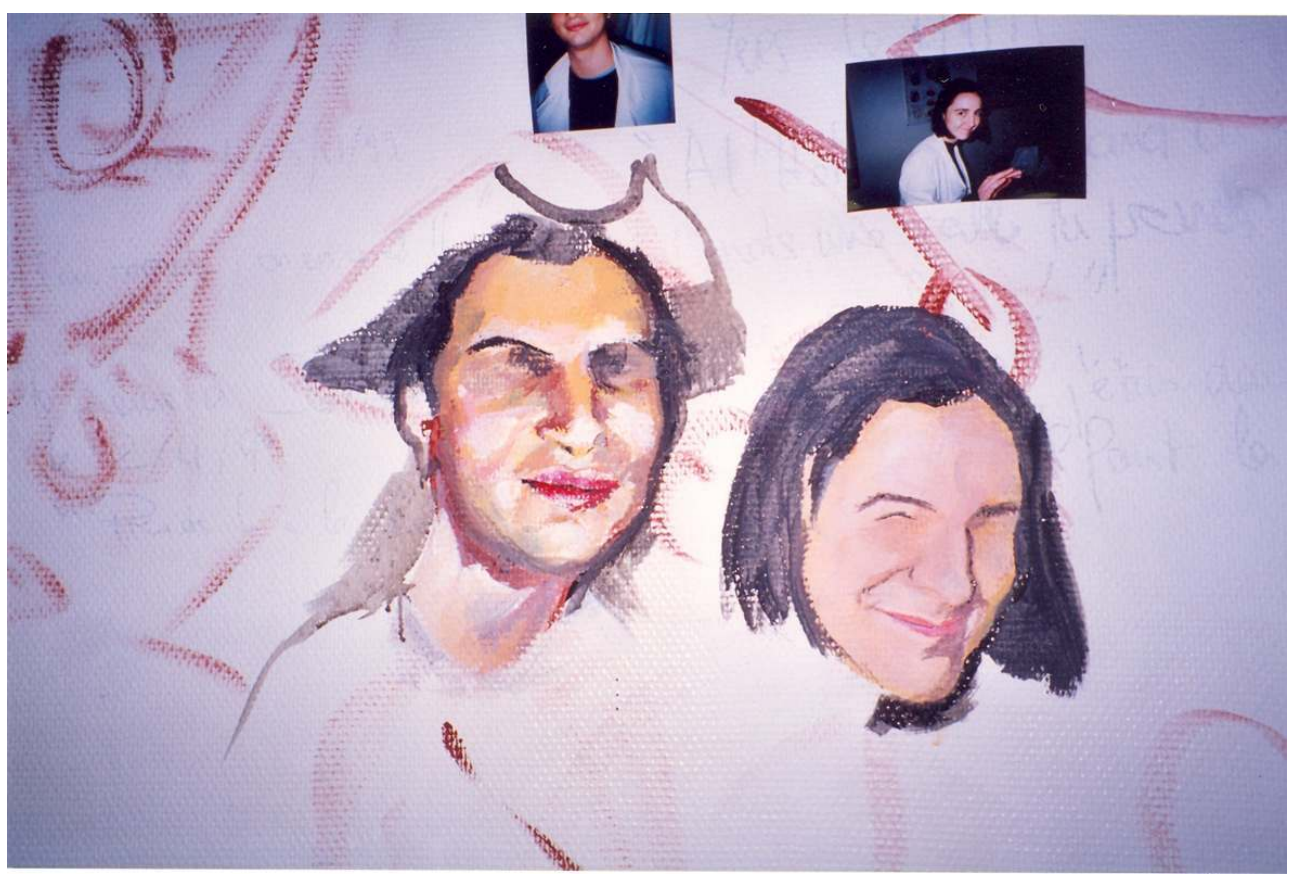

Détail de la réalisation d'un portrait pour la décoration de la salle de garde de l'Hôpital Necker. Paris, Hôpital Necker.

Phot. Christian Hottin, 2000. 


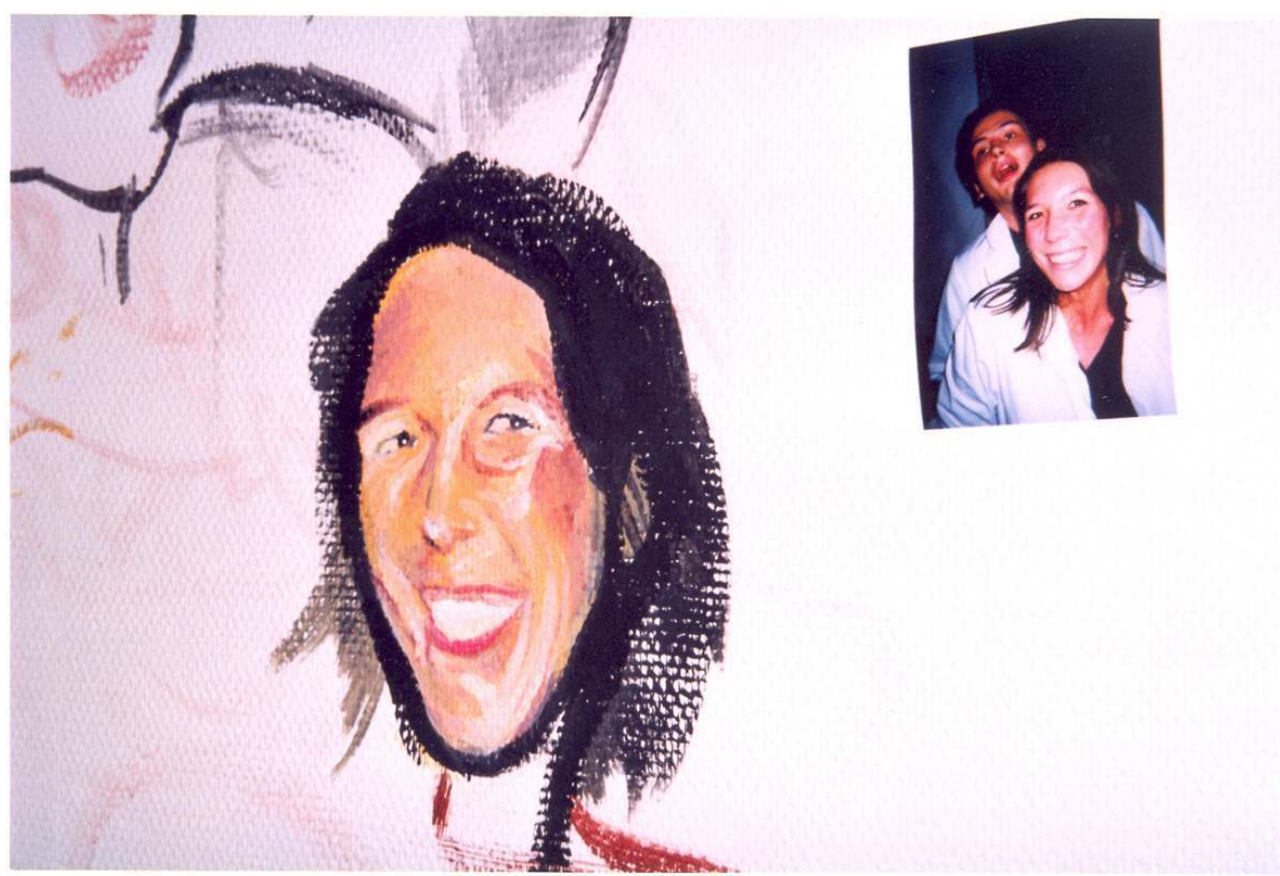

Détail de la réalisation d'un portrait pour la décoration de la salle de garde de l'Hôpital Necker. Paris, Hôpital Necker.

Phot. Christian Hottin, 2000.

13 (fig. $\left.\mathbf{n}^{\circ} \mathbf{1 4}\right)$ (fig. $\left.\mathbf{n}^{\circ} \mathbf{1 5}\right)$ L'artiste travaille pendant les dernières semaines précédant le tonus. Si chaque artiste possède sa propre méthode de travail, il est permis de penser que tous consacrent une part importante de leur temps de travail à la restitution des traits des internes, puisque exécuter un portrait ressemblant est, on l'a vu, une des clauses essentielles du contrat oral qui lie le peintre à l'économe dans cette commande. Dans le cas étudié27, l'artiste, après avoir déterminé la composition d'ensemble de la fresque, commence par dessiner les visages, en s'aidant de photographies qu'il fixe sur le mur pour avoir son modèle sous les yeux. Puis vient l'exécution des corps des personnages, où l'artiste peut donner libre cours à son imagination la plus débridée, ce qui constitue en fait l'application de l'autre clause du contrat, puisqu'une fresque ne saurait être sage. (fig. $\left.n^{\circ} 16\right)\left(\right.$ fig. $\left.n^{\circ} 17\right)\left(\right.$ fig. $\left.n^{\circ} 18\right)\left(\right.$ fig. $\left.n^{\circ} 19\right)$ 
Figure 18

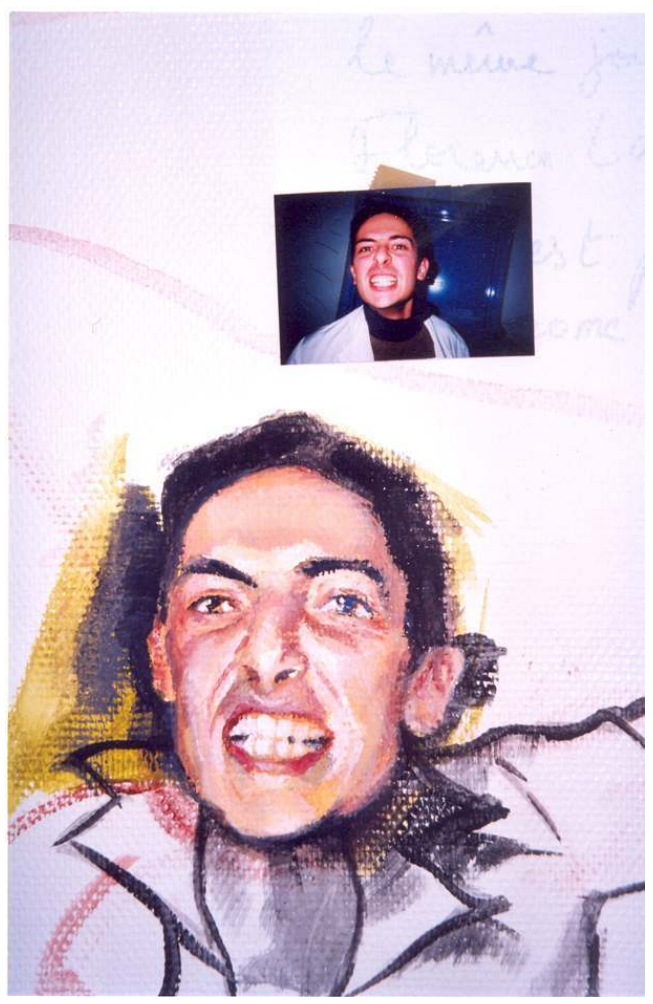

Détail de la réalisation d'un portrait pour la décoration de la salle de garde de l'Hôpital Necker. Paris, Hôpital Necker.

Phot. Christian Hottin, 2000. 
Figure 19

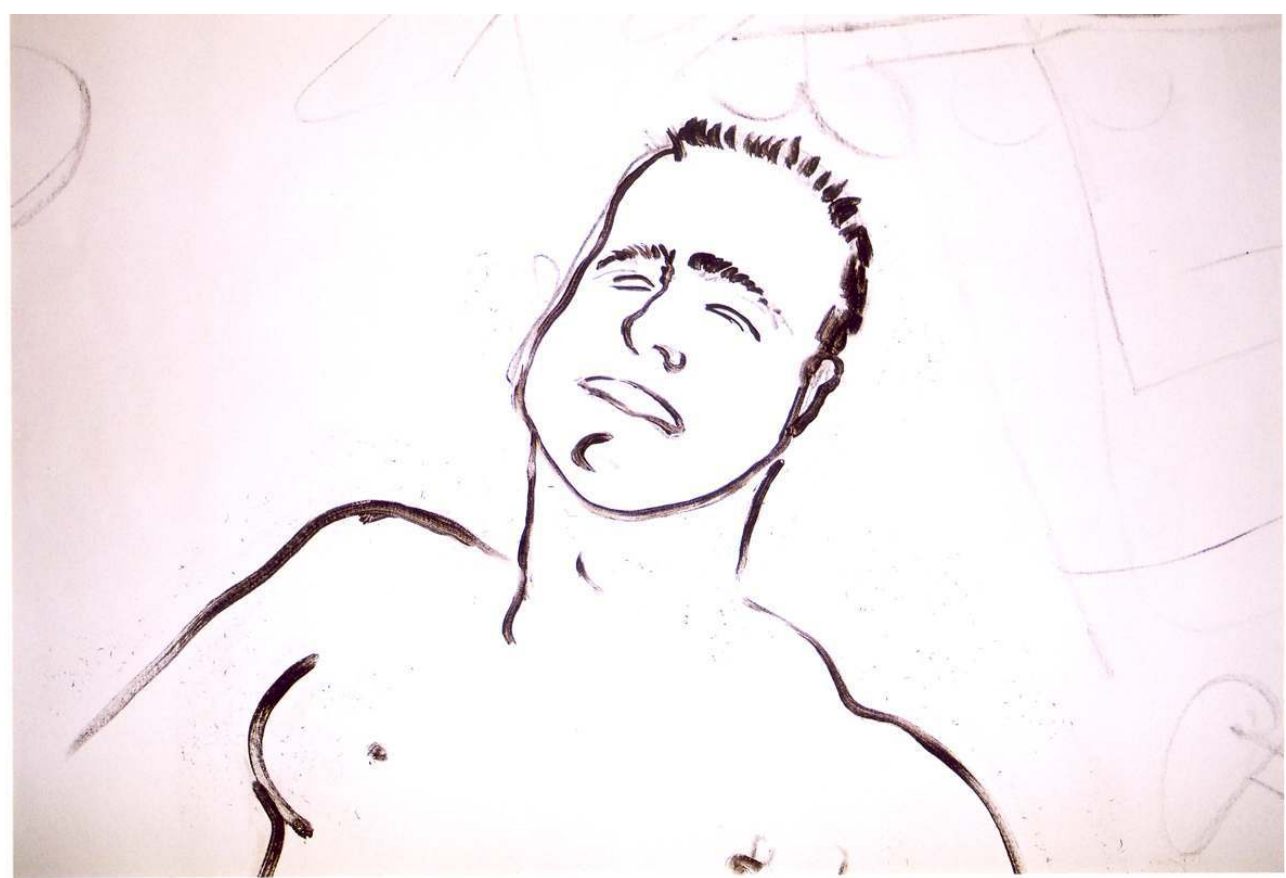

Esquisse pour la réalisation de la fresque de la salle de garde de l'Hôpital Lariboisière. Paris, Hôpital Lariboisière.

Phot. Jean-Christophe Doërr, 2006

Pour garder le secret sur l'œuvre en cours d'élaboration, des tentures la protègent des regards pendant les repas. En effet, la fresque n'est dévoilée que lors du tonus. Elle est alors soumise de fait au jugement de la communauté des internes. Outre le respect du contrat, la qualité esthétique du dessin et l'efficacité humoristique sont, selon les spectateurs et sujets des œuvres, les principaux critères d'appréciation. 
Figure 20

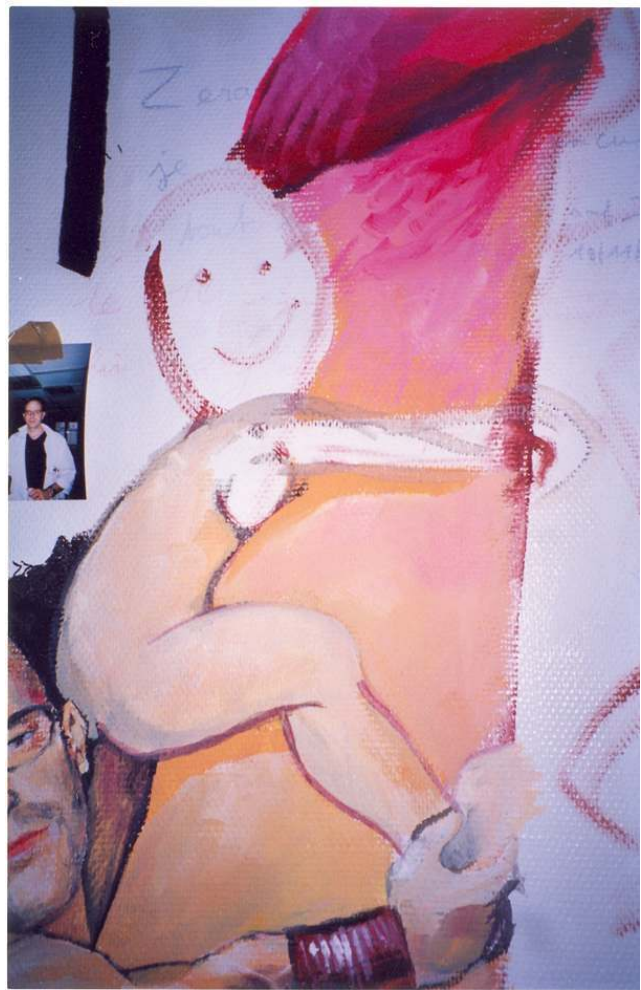

DÉtAIL de LA RÉALISATION DE LA FRESQUE de LA SALLE de gARDE DE L'HôPITAL NECKER. PARIS, HÔPITAL NECKER.

PHOT. CHRISTIAN HOTTIN, 2000. 
Figure 21

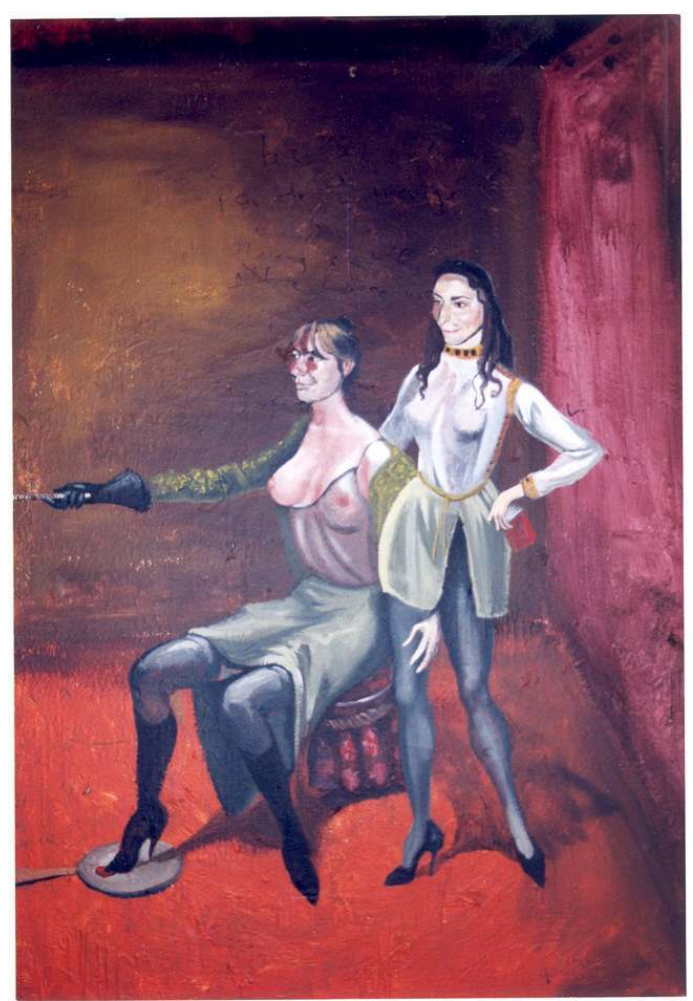

SCÈnE SADOMASOCHISTE, SALLE DE gARDE DE L'ANCIEN HÔPITAL BOUCICAUT. PARIS, HÔPITAL BOUCICAUT.

PHOT. CHRISTIAN HOTTIN, 1998.

Mais qui juge qui ? Il peut sembler évident que l'artiste est en première ligne. Son œuvre est appréciée selon les paramètres cités, qui devraient être eux-mêmes précisément décrits en fonction de paramètres variables ${ }^{28}$. Ainsi, une des normes délimitant l'activité du peintre est la représentation nécessaire de l'acte sexuel, de la façon la plus variée et la plus transgressive possible: sexualité de groupe, pratiques sadomasochistes et mutilations, représentations aberrantes (sexes gigantesques ou multiples) et monstrueuses. Si, à l'intérieur de ce champ, tout est possible, tout n'est pas pour autant permis à l'artiste. À l'occasion des témoignages on voit apparaitre des interdits possibles ou probables dont la transgression est perçue négativement par les membres de la communauté : un peintre évoque par exemple les critiques reçues pour une fresque où les personnages copulaient au milieu des excréments. Plus importante peut-être à analyser est l'assez faible proportion de fresques associant sexualité et représentation de l'acte médical ou chirurgical. Il est en effet très tentant de voir dans cette relative sousreprésentation un équivalent atténué d'un interdit très présent en salle de garde, selon lequel il ne faut ni employer de termes médicaux dans la conversation ${ }^{29} \mathrm{ni}$ introduire d'objet rappelant l'univers médical. (fig. $\left.\mathbf{n}^{\circ} \mathbf{2 0}\right)$ (fig. $\left.{ }^{\circ} 21\right)$ (fig. $\left.\mathbf{n}^{\circ} \mathbf{2 2}\right)$ 
Figure 22

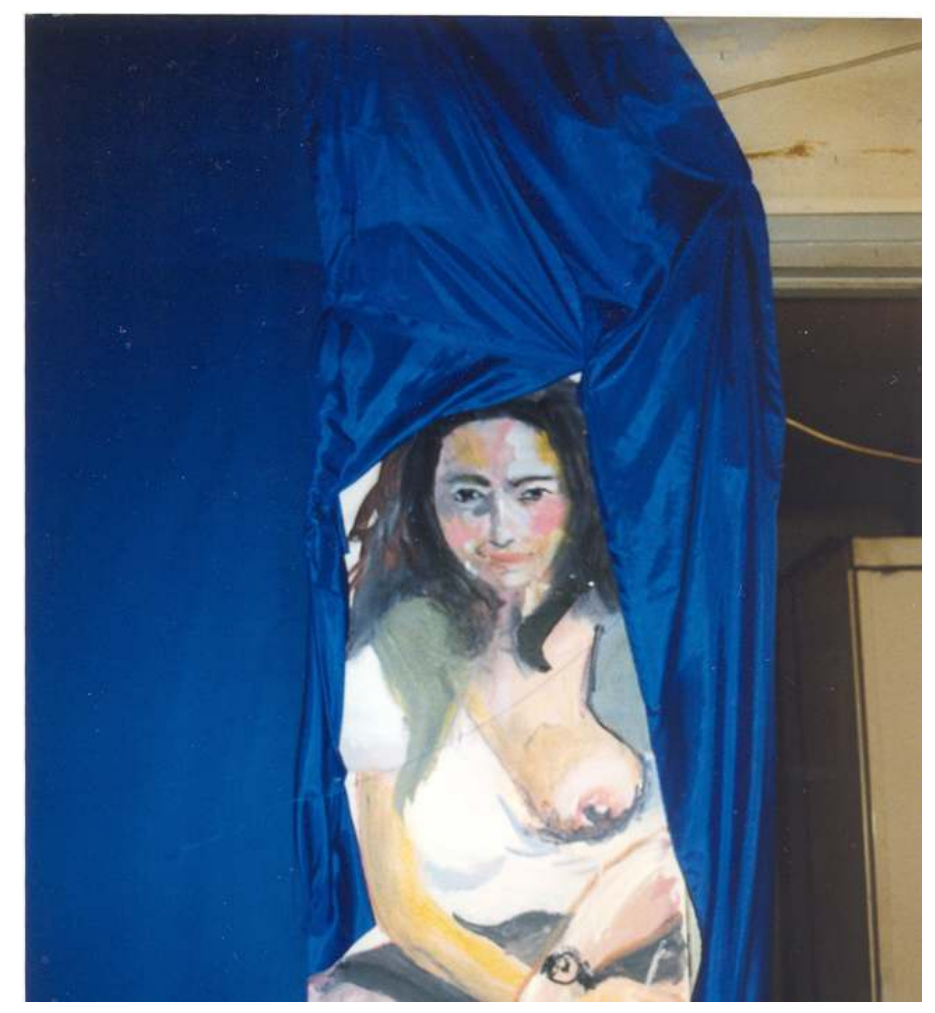

Portrait en pied de l'économinette de la salle de garde de l'Hôpital Bichat. Paris, Hôpital Bichat.

Phot. Christian Hottin, 1998.

Spectateurs de l'œuvre et voyeurs de la scène, les internes en sont aussi le sujet. Donc, face à ce portrait de groupe, ils sont juges, jurés et jugés. Les conseils de l'économe, joints aux photographies, ont permis de composer au physique et au moral le portrait de chacun et de le mettre en scène. La situation de chacun face à l'ensemble du groupe n'est pas neutre, et la fresque peut éventuellement proposer, via le prisme de la sexualité, une image de chacun et des rapports des uns aux autres. Là encore, le récit des réclamations formulées face à certaines représentations jugées dévalorisantes est un indicateur, ténu mais plausible, de l'identification de l'individu à sa représentation.

Quant à l'économe, il est sans doute le plus exposé. En effet, il a reçu des autres internes l'argent nécessaire à l'exécution de la fresque. Il en a discuté le sujet avec l'artiste et lui a fait éventuellement des suggestions. À la différence des autres, il a suivi la genèse de l'œuvre. En tant que commanditaire et en quelque sorte maître d'ouvrage de l'opération, il en assume les chances de succès et les risques d'échec. Les indices de cette perception forte de l'implication de l'économe dans la conduite du projet sont perceptibles avant tout dans certains éléments de la fresque. Certaines compositions réservent une position éminente à l'économe (ainsi dans une transposition de la Cène on pourra lui attribuer la place du Christ) et il est fréquent que son nom figure près de la signature du peintre, la mention du règne personnel venant alors compléter l'indication de la date. Ainsi, sur une fresque de Cochin, une place particulière est attribuée à l'économe et à l'économinette (sur une porte), tandis que leurs noms accompagnent les dates du semestre: «Sous le règne de Ève Kollar et Laurent Lavau mai-octobre $1992 »$. 
La fresque fait donc partie des éléments qui permettent au cours du semestre d'évaluer l'action de l'économe et qui in fine valident ou invalident la confiance mise en lui par ses collègues lors de sa désignation. L'organisation du tonus est un autre de ces indicateurs, mais le plus important est sans aucun doute la capacité de l'économe à jouer son rôle d'organisateur, de régulateur et de censeur dans la vie quotidienne de la salle de garde ${ }^{30}$. De même que la représentation de l'interne sur la fresque peut être ressentie comme une appréciation portée sur l'individu, de même l'aptitude de l'économe à gérer sa salle de garde (on peut en effet dire qu'il en administre l'économie, et ce terme recouvre alors l'ensemble des rapports humains à l'œuvre dans la vie collective au cours du semestre et non la seule gestion des contributions financières des internes) est un élément qui contribue à façonner pour le groupe la perception globale de celui qui a été choisi comme chef. À ce dernier de se conformer en acte à l'image idéale de beau parleur sachant se faire respecter que véhicule la littérature produite par les «fossiles » sur l'internat: " [L'économe] hermétique à la critique, craint pour son autorité, qui n'est que calembours et promptitude aux répliques, il en appelle à la répartie moqueuse, à la satire impitoyable, et il est parfois vain de lui résister jusqu'au café $»^{31}$. Dans le contexte spécifique de la vie en salle de garde, images, rites et rôles de chacun paraissent être autant de paramètres qui contribuent à construire pour le groupe l'identité et la personnalité de chaque membre, en parallèle - mais en lien ? - avec le travail hospitalier ${ }^{32}$.

\section{Origines d'une collaboration}

Commander une fresque ne répond donc pas au seul désir d'égayer de scènes gaillardes ou galantes les murs d'une salle de restauration collective : ce processus finement codifié s'inscrit dans la temporalité interne de la communauté. La réussite du projet et les représentations figurées qui en découlent tiennent leur place dans le jeu complexe d'évaluation du chef par le groupe qui l'a désigné et d'appréciation réciproque de chaque membre de la salle par tous les autres. L'inscription de cette pratique collective dans le temps, et singulièrement le discours produit par les historiographes de la communauté sur son ancienneté, montrent cependant que la fresque ne contribue pas seulement à définir l'identité des internes dans le cadre restreint et éphémère d'un semestre de garde: la fresque prend place, comme un maillon important, dans la chaîne de représentations qui, en mettant en évidence la proximité entre médecins et artistes, contribue à la définition des internes comme institution.

Historiens et chroniqueurs de l'Internat insistent volontiers sur l'ancienneté de la pratique de la fresque, perçue avec les chansons comme l'une des plus anciennes traditions de la salle de garde ${ }^{33}$. Tous évoquent celle de l'ancienne Charité comme étant l'exemple le plus ancien et le plus illustre d'une longue lignée de fresques. Souvent gravée et glosée, cette salle couverte de fresques par Gustave Doré et d'autres peintres semble avoir été perçue dès le XIX ${ }^{e}$ siècle comme un espace particulièrement digne d'intérêt : sa confiscation au profit des professeurs pour en faire une salle réservée à leur usage peut être regardée comme une insertion précoce $d u$ lieu dans le patrimoine collectif du groupe. On attribue alors aux internes une nouvelle salle, dans un ancien local des chapelains. D'autres artistes, dont Bellery Desfontaines, la décorent aussi richement que la première. Lorsque la destruction de l'hôpital est décidée, dans le but de construire une nouvelle faculté de médecine, toutes les peintures sont déposées et les salles de garde reconstituées au Musée de l'APHP ${ }^{34}$. Leur transformation en objet muséal (en grande 
partie, il est vrai, en raison de la qualité des peintres qui y travaillèrent) est un indice du fort attachement de la communauté à ce lieu, regardé comme le paradigme de la salle de garde et comme le décor idéalisé de la vie qu'on y mène.

La description détaillée de ces fresques anciennes s'accompagne nécessairement de la liste exhaustive des artistes qui œuvrèrent sur place ${ }^{35}$, ainsi que d'une présentation des modalités de leur exécution qui tend à apparaître très différente du processus de commande que nous avons précédemment observé : dans cette salle de garde mythique, lieu d'expression artistique, l'interne se pose en mécène, il offre le gite et le couvert à l'artiste maudit et démuni, qui paie en nature musicale, littéraire ou picturale. En effet, tous les arts sont mis à contribution pour renforcer cette représentation de la salle de garde en lieu d'expression culturelle. On évoque Verlaine à Broussais, Toulouse-Lautrec et Vuillard à l'Hôtel-Dieu ${ }^{36}$. Ailleurs, le petit-fils de Watteau est mentionné à Issy-lesMoulineaux, tandis que Toulouse-Lautrec, cette fois-ci, « dîne à Saint-Louis $\|^{37}$. On fait encore allusion, à Laënnec, à une fresque de Puvis de Chavannes, «plus audacieuse que celle du Panthéon». Plus près de nous, et selon des sources sans doute plus aisées à vérifier, Foujita est répertorié comme « interne d'honneur » de Vaugirard. Les chansons de salle de garde jouissent des mêmes nobles origines, alors que la vérification de l'authenticité des attributions est encore plus hasardeuse ${ }^{38}: L a$ colonne Vendôme à Théophile Gautier, Minette et Soixante-neuf à Maupassant, ou encore Les filles de Loth à Alfred de Musset ${ }^{39}$. Dans des ouvrages qui offrent une représentation de l'histoire de l'institution vue de l'intérieur, totalement dépourvue de distanciation critique et de démarche d'analyse réflexive, la salle et ses fresques sont des éléments essentiels de la description de la "vie quotidienne de l'Internat", tranches de vie qui succèdent habituellement aux récits linéaires et fastidieux des étapes de l'évolution institutionnelle de la communauté.

À mettre en parallèle avec cette évocation récurrente des liens qui unissent grandes figures littéraires ou artistiques de la bohème parisienne et carabins, une collaboration intense et fructueuse entre artistes et médecins existe et se manifeste de manière concrète. Elle mérite d'autant plus l'attention qu'elle est particulièrement vivace lors de l'un des moments qui occupe une place centrale dans le temps interne de la communauté. La collectivité des internes, généralement éclatée et dispersée en différents lieux, soumise au cycle perpétuel des semestres que scandent les tonus, s'ancre dans le cycle plus large, puisque annuel, du temps universitaire par le biais du concours, suite d'épreuves singulièrement ritualisées, que la représentation graphique et la rumination littéraire ${ }^{40}$ contribuent à ériger en temps fort de la vie collective, en plus d'être l'acte fondateur de la naissance de l'interne en tant que membre de l'institution. Événement strictement individuel, placé dans le temps à l'autre extrémité de la vie de l'interne, après la succession des semestres, l'enterrement est une autre occasion de réjouissances.

Dans l'entre-deux, seul moment du temps universel à pouvoir être revendiqué par la communauté entière comme une expression collective de son identité, le bal annuel assume d'autant plus cette fonction que, par sa localisation dans un lieu public et par le défilé qui l'accompagne, il est un des rares moments où le groupe tout entier se rend totalement visible pour tous ses membres et pour tous ceux qui lui sont étrangers. Il est l'occasion de cette collaboration intime entre l'artiste et l'interne. Les peintres et les dessinateurs sont sollicités pour mettre au point l'affiche du bal, ainsi que les cartons d'invitation (en général ils sont différents selon qu'ils sont destinés aux hommes ou aux femmes). D'autres, en particulier les sculpteurs ou les décorateurs, participent à la 
réalisation des chars du défilé, qui tous doivent illustrer un thème (en général médical et sexuel). Bien que bal et défilé concernent une époque plus ancienne que celle des fresques analysées plus haut (l'âge d'or du bal se situe avant 1950), on y retrouve la même omniprésence de la thématique sexuelle (les maladies vénériennes sont bien représentées, et les programmes décoratifs conçus pour les chars ne sont pas sans ambiguïté, bien que souvent empruntés à l'actualité médicale ou politique: «La pénétration pacifique et antiphysique au Maroc" ou encore "La vérole aux ailes de chauve-souris agonise une aiguille meurtrière et démesurée plantée en son sein (...) Monneyrat arrive trop tard, une seringue inutilisée à la main, et dispute à Herlich le trophée de la victoire »). Tout comme dans le processus de monstration des fresques, on retrouve dans la présentation des chars, qui sont désignés par le nom des différents hôpitaux, le principe de l'évaluation d'une partie de la collectivité par l'ensemble de celleci : les critiques de la presse médicale commentent, comparent les chars, désignent les salles de garde qui ont accompli le travail le plus remarquable et stigmatisent les moins inventives ${ }^{41}$.

À plusieurs reprises, est évoquée avec précision la fructueuse collaboration des arts et de la médecine :

«Toutes les imaginations ont fait merveille et en retour chacun a été émerveillé. Aimable et juste récompense de nombreux travaux préalables auxquels se sont livrés laborieusement internes et externes, aidés par leurs amis les peintres (...) Voici [sont reproduites des photographies montrant la préparation des chars] qui représente ces gais travaux en cours d'exécution. N'appelle-t-il pas le sourire, cet affairement de nos carabins et rapins, devenus frères sous la blouse blanche protectrice? $»^{42}$

Et le même critique dans un autre compte rendu mentionne encore plus précisément ces liens :

«Toute salle de garde conserve un peu des éléments de Montmartre ou de Montparnasse. Les internes sont les plus charmants, les plus délicats des amphitryons, et les artistes les convives les plus joyeux. C'est plaisir de voir quelle entente cordiale s'établit entre l'extrême science et la fantaisie débordante.

Jamais de discussion désobligeante entre internes et rapins : ils ont à peine pris contact qu'ils se trouvent en sympathie. D'une telle collaboration il ne peut naître que projets originaux et qu'organisation de fêtes dans une note pittoresque et amusante.

(...) Gaston Trilleau le peintre graveur nous fit nombre de croquis aussi spirituels que gaillards. Internes et artistes se renvoyaient les mots amusants, les idées drôles.

(...) La gestation du projet s'opéra au milieu d'éclats de rire $»^{43}$

L'article est copieusement illustré : dessins montrant les chars des différents hôpitaux, reproductions de cartes d'invitations, gravures présentant les bannières (également conçues par des artistes, dont André Henry, l'auteur de la chronique). L'artiste, élément extérieur au groupe bien que potentiellement très impliqué dans la préparation du bal, comme c'est le cas pour Henry, n'est pas le seul à juger et jauger à l'aune des fresques les différentes salles de garde et le groupe des internes dans son ensemble: un «fossile » apporte sa contribution, fort critique au demeurant, en manifestant son désaccord avec les orientations esthétiques du moment ${ }^{44}$. La collaboration s'étend donc à l'appréciation critique portée sur les réalisations collectives des rapins et des carabins: les membres anciens du groupe évaluent le travail artistique de leurs successeurs, tandis qu'un peintre peut exprimer son opinion et faire part de son expérience de la conception des chars dans la presse spécialisée médicale. En plus de leur contribution matérielle à la réalisation des 
chars, les internes apportent une dimension supplémentaire à leur intervention dans la sphère artistique en composant les textes « littéraires » qui servent de commentaire au défilé, comme en témoigne cet extrait qui accompagne la présentation du char de l'Hôtel Dieu, dédié au thème de la Foudrothérapie :

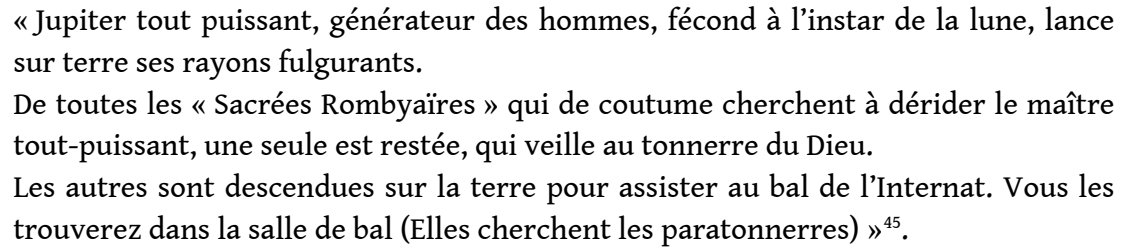

27 À travers la préparation de ce moment festif et crucial qu'est le bal annuel de l'Internat, on voit donc s'esquisser une nouvelle forme de rapprochement entre le peintre et l'interne, fondée non plus sur le principe de la commande et du mécénat (réel ou supposé), mais reposant sur l'idée d'une parenté et d'une égalité entre les deux groupes, égalité symbolisée par la blouse commune que mentionne Henry dans une de ses chroniques. De l'égalité à l'identité, reste un pas à franchir : que l'interne à son tour devienne artiste et crée l'œuvre qui contribuera à le définir et à donner une image de sa communauté tout en témoignant de son insertion dans le groupe.

\section{Figures d'une identification}

Artistes, certains médecins le sont assurément. Au point de réaliser eux-mêmes des fresques pour les salles de garde. Cette implication directe de membres de la communauté dans la production de représentations iconiques collectives n'est pas propre au monde de l'Internat : on a souligné précédemment que le cas s'était produit à l'École Centrale, à l'École Polytechnique ${ }^{46}$ et bien entendu dans les écoles d'arts ${ }^{47}$. Des fresques exécutées par des internes sont attestées dès le siècle dernier. Jacques Le Pesteur mentionne celle que peignit Paul Richer en 1877 à l'ancienne Pitié ${ }^{48}$, et, sans lui attribuer explicitement la composition de décors peints, il évoque également le nom de Farabeuf, qui fut un extraordinaire dessinateur d'anatomie ${ }^{49}$. Plus près de nous, un exemple célèbre de fresque peinte par un interne est donné par l'extraordinaire composition exécutée pour le plafond de la salle de l'hôpital Saint-Antoine : dans la salle qui accueille déjà une des fresques « historiques » les plus fameuses (La guerre de Gaules datée de 1945), les photos encadrées des internes du semestre sont collées au plafond sur tout le tour de la pièce. L'espace central du plafond est divisé en deux parties juxtaposées : d'un côté, une scène de partouze classique, mais exécutée en bas-relief, de l'autre une table et des chaises fixées directement au plafond et reproduisant par un effet de miroir le mobilier de la pièce et le repas qui s'y déroule quotidiennement. Sur la table figure cette légende « (...) Verneaux un économe renversant $»^{50}$. Outre son aspect spectaculaire, cette œuvre retient l'attention par le traitement original qu'elle donne à voir d'une thématique topique, puisque sexualité de groupe et portrait collectif, au lieu d'être confondus en une œuvre unique, y sont traités séparément. En outre, de même qu'on ne manque pas de signaler les internes auteurs de fresques, on se plaît à rappeler que de nombreux médecins ont également exercé avec talent l'un ou l'autre des arts, soit pour en faire leur activité principale (ainsi le romancier Georges Duhamel), soit pour en jouir comme d'un violon d'Ingres (comme Henri Mondor). 
Pour spectaculaires ou plaisantes qu'elles soient, ces manifestations du sens artistique des internes n'en demeurent pas moins exceptionnelles. Plus intéressant, bien que plus difficile à saisir, apparaît l'ensemble des comportements, gestes ou attitudes propres à la salle de garde qui, bien que ne relevant pas des arts, implique l'art. Cet art qui n'appartient pas aux arts, il se manifeste par l'habileté technique, qu'elle soit manuelle ou rhétorique, et s'évalue par le jugement du groupe. Un grand nombre de situations en salle de garde fonctionnent comme autant d'injonctions à faire preuve d'habileté, avec, comme sanction, le gage symbolique qui traduit le jugement réprobateur porté par les autres membres du groupe. Il en va ainsi de l'aptitude à respecter les interdits verbaux propres à la salle de garde, tels que l'obligation d'utiliser des périphrases pour désigner les termes médicaux techniques, qui sont proscrits pendant toute la durée du repas. De même, l'habilité manuelle se manifeste avec éclat dans l'ouverture des bouteilles de vin : l'usage du tire-bouchon étant interdit en salle de garde, force est de déboucher les bouteilles avec un simple couteau, en sciant précautionneusement le goulot ${ }^{51}$. Pour décrire toute la minutie déployée lors de cette opération, Patrice Josset renvoie explicitement à la pratique de l'artisanat:

«Comme tout travail relevant d'un apprentissage, l'habileté manuelle couplée à la compréhension intellectuelle du geste aboutissent à la réalisation de l'acte parfait. L'épreuve peut paraître dérisoire, petite, sans importance ; en fait elle est cruciale dans sa simplicité. Comme le tailleur de pierre doit savoir explicitement où frapper pour fendre la pierre, la tailler, la sculpter, l'apprenti et surtout le compagnon en salle de garde doivent savoir où entamer le verre de la bouteille pour le fendre habilement $»^{52}$.

C'est surtout pour « le compagnon » que la manifestation de cette habileté est impérative. Encore une fois, le comportement de l'un est l'objet du jugement et de l'évaluation de tous les autres. Si les situations varient sans cesse, relevant chaque fois d'un rite ou d'un usage différent (ouverture de bouteille, commande de fresque, esprit de répartie), toujours se dessine, derrière le masque souriant de la blague de carabin, le regard omniprésent des pairs. Cette obligation d'être le plus habile, le plus adroit, le plus brillant, apparait une dernière fois dans toute son importance lors de l'enterrement, au moment où l'interne doit prononcer son discours. "Alors on jouait toute sa carrière ", rapporte un fossile ${ }^{53} . .$. et l'habileté peut parfois revêtir des formes insolites :

"Un chirurgien, lors de son enterrement, a impressionné tout le monde. $\mathrm{Au}$ moment de dire son texte, il s'est mis debout, a ouvert sa braguette et a pissé pendant toute le durée de son discours. Je peux vous dire qu'elle a été longue sa réponse. Je n'ai jamais vu pisser aussi longtemps ${ }^{54}$.

31 Hors du contexte précis de l'exécution des fresques, mais toujours en lien avec elles (puisque la fresque n'est qu'une des modalités d'expression de cet impératif d'autoévaluation collective qui régit la vie en salle de garde, tandis que des situations, qui ne relèvent pas a priori de l'art, $\mathrm{y}$ font appel - $\mathrm{y}$ compris à la manière des performances de l'art contemporain), multiples sont donc, tant dans la dispersion diachronique que dans la diversité des pratiques et des réalisations, les liens qui unissent l'interne à l'art et aux artistes.

Si elle se présente comme une parenthèse temporelle dans la journée d'hôpital, comme un lieu affranchi des règles de cette institution et possédant sa mesure du temps particulière autant que son propre système de règles de vie, les parois qui séparent la salle du monde extérieur sont trop minces pour ne pas voir dans le jeu qui s'y joue la poursuite, par d'autres moyens et avec d'autres mots (ceux qui ne sont pas interdits en 
salle...) des relations interpersonnelles d'émulation et de compétition qui opposent les différents membres de l'institution au sein du champ des études médicales et de la vie hospitalière.

\section{Conclusions}

Il faut l'avouer : telles qu'elles sont, parlant plus aux sens ou aux souvenirs, ces fresques n'encouragent guère l'analyse. Pour le médecin, l'ancien interne qui se fait l'historiographe de la corporation, elles servent de point d'ancrage privilégié à l'évocation de l'univers de la salle de garde. Pour l'artiste, le photographe, il y a là la matière d'un ouvrage gai et coloré, dans lequel chaque page offre au lecteur une occasion de rire ou de s'étonner ${ }^{55}$. A contrario, de la démarche initiée ici, deux points semblent pour le moment à retenir. Le processus de commande des fresques de salle de garde obéit à des règles précises et complexes bien que non écrites. Par bien des traits, ce processus est comparable à celui observé lors de la commande d'œuvres par les membres d'autres institutions, qu'il s'agisse de peintures « officielles » ou non. Ce processus est sous-tendu par des motivations collectives plus riches que le simple désir de décorer un espace de la vie commune, et il revêt une importance toute particulière pour l'économe qui dirige la salle de garde. En outre, la réalisation des fresques est une pratique ancienne. L'étude de la production de décor au XIXe siècle et l'analyse des conditions de création d'artefacts proches des fresques (bannières, cartes, char de bal) mettent en évidence les liens de collaboration entre carabins et artistes et un certain désir d'identification à ces derniers. Là encore, à travers la création d'œuvres, un certain nombre de caractères que le groupe souhaite intégrer à son identité apparaissent.

De la même manière que les conditions de production des œuvres, leurs thématiques (portrait de groupe, expression de la vie sexuelle) et leur place dans la vie quotidienne de la salle fournissent un éclairage sur la communauté des internes: tels seront les développements ultérieurs de cette étude ${ }^{56}$.

\section{NOTES}

1. Voir : VAISSE, Pierre. La troisième république et les peintres. Paris : Flammarion, 1995. Voir aussi : HOTTIN, Christian. «Les commandes de peintures » et «Les collections de bustes ». Universités et grandes écoles à Paris, Les Palais de la Science. Paris : AAVP, 1999, p. 59-67 et 68-74.

2. Le résultat de ce travail a été publié : HOTTIN, Christian. Quand la Sorbonne était peinte. Paris : Maisonneuve et Larose, 2001.

3. Un des exemples les plus représentatifs de ce type d'œuvres est la peinture de la thurne François-Poncet à l'École Normale Supérieure. La mise à l'épreuve de l'hypothèse de travail a été développée dans un article à travers la mise en rapport de cette peinture avec le monument aux morts de l'École : HOTTIN, Christian. « 80 ans de la vie d'un monument aux morts, Le monument aux morts de l'École Normale Supérieure ». Labyrinthe, n 5, hiver 2000, p. 61-79. 
4. Le terme de fresque sera employé par commodité, ce qui est recevable dans la mesure où cette dénomination technique erronée est d'un usage courant pour désigner les peintures des salles de garde.

5. De nombreux recueils existent, souvent dactylographiés et parfois publiés. Un parmi d'autres : Le Bréviaire du carabin. Strasbourg : Association Amicale des Étudiants en Médecine de Strasbourg, 1981. Voir aussi : http://www.leplaisirdesdieux.com/LePlaisirDesDieux/LES.SALLES.DE.GARDE/ LES.PAILLARDES/vospaillardes.html.

6. Voir : JOSSET, Patrice. La salle de garde, Histoire et signification des rituels des salles de garde de médecine, chirurgie et pharmacie, du Moyen-Âge à nos jours. Paris : Le Léopard d'Or, 1996.

7. Une collection très complète est conservée à la Bibliothèque nationale de France, Département des estampes et de la photographie. Voir aussi : Cartes de bal de l'Internat en Médecine et Chirurgie des Hôpitaux de Paris. CBUF, Grenoble, [1985].

8. Le fossile est un ancien interne qui fréquente encore la salle de garde.

9. Voir: FOSSARD, Jacques. Histoire polymorphe de l'internat en médecine et chirurgie des hôpitaux et hospices civils de Paris. CPBF, Grenoble, 1981.

10. Voir : JOSSET, Patrice. La salle de garde, Histoire et signification des rituels des salles de garde de médecine, chirurgie et pharmacie, du Moyen-Âge à nos jours. Paris : Le Léopard d'Or, 1996, p. 25.

11. Pour un témoignage sur la vie en salle de garde au début du $\mathrm{XX}^{\mathrm{e}}$ siècle, voir : QUENU, Jean. Notre Internat. Paris : Doin, 1971.

12. Voir : "Interview du professeur François Lhermitte». Dans BALLOUL, Patrick. La salle de garde ou le plaisir des dieux. Urrugne : éd. de Loya, 1993, t. I, p. 50-52.

13. Il s'agit du trône de l'Économe, généralement en forme de phallus et de la roue qui sert à désigner les sanctions encourues par ceux qui ont enfreint le règlement de la salle.

14. L'initiation sentimentale, vers 1887 - crayon de couleur et aquarelle, 29,2 x 18, 2. Paris, Musée du Louvre, département des arts graphiques. Inv RF 5265. Reproduction : Le cabinet des dessins: Rops. Paris : Flammarion - musée galerie de la Seita, 1998, p. 106-107.

15. Ces fresques ainsi que les suivantes sont visibles sur le site des internes des hôpitaux de Paris à l'adresse suivante : http://www.leplaisirdesdieux.com/LePlaisirDesDieux/ LES.SALLES.DE.GARDE/FRESQUESDG/Bichat.html. D'après les légendes des photographies, ces fresques ont été exécutées en 1964 par des élèves de l'École des Beaux-Arts.

16. Visite effectuée le 26 janvier 1998, alors que Dilek Sapmaz était économinette de Bichat.

17. Tonus : "réunion d'internes se déroulant généralement le soir en fin de semestre ». JOSSET, Patrice. La salle de garde, Histoire et signification des rituels des salles de garde de médecine, chirurgie et pharmacie, du Moyen-Âge à nos jours. Paris : Le Léopard d'Or, 1996, p. 215.

18. Économe : "L'économe représente l'autorité de la salle de garde. Il en a l'autorité royale ». JOSSET, Patrice. La salle de garde, Histoire et signification des rituels des salles de garde de médecine, chirurgie et pharmacie, du Moyen-Âge à nos jours. Paris : Le Léopard d'Or, 1996, p. 203. Patrice Josset souligne les similitudes de rites et de compétences qui existent entre l'économe et le Vénérable Maître d'une loge maçonnique.

19. Roue: "La roue sert à fixer les taxes de ceux qui ont fait une faute en salle de garde ". JOSSET, Patrice. La salle de garde, Histoire et signification des rituels des salles de garde de médecine, chirurgie et pharmacie, du Moyen-Âge à nos jours. Paris : Le Léopard d'Or, 1996, p. 212.

20. Selon les cas, l'économinette est l'assistante féminine de l'économe ou l'économe en titre de sexe féminin.

21. Entretien avec Patrice Josset, 9 décembre 1997.

22. Entretien avec Grégory Makles, peintre des fresques de salles de garde, 22 avril 2000.

23. http://www.leplaisirdesdieux.com/LePlaisirDesDieux/LES.SALLES.DE.GARDE/FRESQUESDG/ fresques.html. Le site internet de l'Association des salles de garde conseille aux économes de contacter la grande masse des beaux-arts et donne l'adresse de Grégory Makles. 
24. Entretiens avec Ève Kollar (ancienne économe de Cochin) le 10 février 1998 et avec Grégory Makles le 22 avril 2000. C'est le peintre qui mentionne les prix les plus bas, mais il évoque également une fresque inachevée pour une salle de garde de pharmacie et commandée 15000 francs.

25. Jacques le Pesteur souligne cette mission en qualifiant l'économe de « conseiller technique pour la mise en scène" de la fresque. LE PESTEUR, Jacques. Fresques des salles de garde. Paris : Ramsay, p. 43.

26. Qu'elles soient artistes, internes ou fossiles, toutes les personnes interrogées sont d'accord sur ce point ; comme le montrent les entretiens avec Ève Kollar, Patrice Josset et Grégory Makles. Afin de s'assurer de la ressemblance, l'économe est tenu de fournir des photographies de ses camarades à l'artiste. Cette prescription vaut aussi pour les dîners de patrons. Voir : LE PESTEUR, Jacques. Fresques des salles de garde. Paris : Ramsay, p. 43.

27. Entretien avec Grégory Macklès lors de la réalisation d'une fresque à l'hôpital Necker le 22 avril 2000.

28. Il ne s'agit que d'une simple hypothèse, mais il est probable que l'appréciation de la valeur esthétique de l'œuvre s'effectue en fonction des productions imagières les plus répandues du moment (bandes dessinées pour adultes, caricatures).

29. Voir : JOSSET, Patrice. La salle de garde, Histoire et signification des rituels des salles de garde de médecine, chirurgie et pharmacie, du Moyen-Âge à nos jours. Paris : Le Léopard d'Or, 1996, p. 41-49. Les interprétations possibles de cet interdit seront envisagées ultérieurement. Patrice Josset propose en l'occurrence un équivalent avec les pratiques maçonniques, le mot interdit étant alors un mot sacré, qui doit donc être caché au profane éventuellement présent en salle de garde.

30. Patrice Josset évoque les sanctions encourues par un économe qui ne se montre pas à la hauteur de sa fonction, ainsi la «battue " (manière particulière de frapper sur la table en cadence) dite "merdique " qui «salue un événement lamentable, plat détestable, prestation lamentable d'un économe (...). Un économe agira toujours au mieux pour éviter de recueillir une battue de ce genre ». JOSSET, Patrice. La salle de garde, Histoire et signification des rituels des salles de garde de médecine, chirurgie et pharmacie, du Moyen-Âge à nos jours. Paris : Le Léopard d'Or, 1996, p. 84.

31. LE PESTEUR, Jacques. Fresques des salles de garde. Paris : Ramsay, p. 25.

32. L'enterrement (cérémonie qui marque la fin des années d'internat) d'un interne, et en particulier le discours prononcé lors de cette cérémonie, jouent un rôle semblable.

33. LE PESTEUR, Jacques. Fresques des salles de garde. Paris : Ramsay. Voir aussi : FOSSARD, Jacques. Histoire polymorphe de l'internat en médecine et chirurgie des hôpitaux et hospices civils de Paris. CPBF, Grenoble, 1981.

34. Sur ces œuvres, voir: "Salon d'un carabin devenu mandarin». Catalogue des collections permanentes du Musée de l'assistance Publique des Hôpitaux de Paris. Paris, p. 140-145.

35. FOSSARD, Jacques. Histoire polymorphe de l'internat en médecine et chirurgie des hôpitaux et hospices civils de Paris. CPBF, Grenoble, 1981, p. 40.

36. LE PESTEUR, Jacques, op. cit., p. 19.

37. FOSSARD, Jacques, op. cit., p. 39.

38. En regard de cette appropriation du monde littéraire par le monde médical, il faudrait aborder, mais cela dépasse le cadre de cette étude, toute la question de la fascination exercée par la médecine, les internes et, plus précisément, les salles de garde sur le monde de la création littéraire. La salle de garde apparaît en effet dans de nombreux romans, tels que ceux des Goncourt, de Paul Bourget ou de Pierre Very, sans parler des œuvres écrites par des médecins (La Pierre d'Horeb de Georges Duhamel).

39. LE PESTEUR, Jacques, op. cit., p. 39. 
40. On reviendra sur la place du concours dans la construction de l'identité collective. Voir, parmi d'autres textes : FOSSARD, Jacques, op. cit., p. 89-91 et illustrations p. 49 et p. 117.

41. Ainsi, André Henry critique « les hôpitaux, pourtant de grande taille, qui n'ont pas fait de chars, alors que le modeste Hospice des ménages [Corentin Celton à Issy-les-Moulineaux] en a un ». Voir : HENRY, André. «Le bal de l'Internat 1911 ». La Chronique médicale, 1911, p. 705-717, p. 716.

42. HENRY, André. « Le bal de l'Internat 1911 ». La Chronique médicale, 1911, p. 707-717, p. 705.

43. HENRY, André. « Le bal de l'Internat 1910 ». La Chronique médicale, 1910, p. 736-738, p. 738 et 739.

44. «Impressions d'un fossile sur le bal de l'Internat de 1910 ». La Chronique médicale, 1910, p. 786-788.

45. HENRY, André. « Le bal de l'Internat 1910 ». La Chronique médicale, 1910, p. 736-738, p. 740.

46. Dans un contexte différent de celui des salles de garde, mais qui mobilise également les membres de la communauté dans un processus d'évaluation réciproque, les élèves composant les différentes listes en concurrence pour l'élection du bureau de la promotion réalisent lors des « campagnes-kès» de grandes fresques peintes sur les murs des logements étudiants de l'École. Voir: HOTTIN, Christian. Quand la Sorbonne était peinte. Paris: Maisonneuve et Larose, 2001, p. 260-261.

47. Voir : HOTTIN, Christian. Quand la Sorbonne était peinte. Paris : Maisonneuve et Larose, 2001, p. $148-152$.

48. Le cas de Paul Richer est à ce titre doublement intéressant, puisque cet ancien interne, également sculpteur, exécuta en 1910 le monument funéraire du professeur Victor Cornil, aujourd'hui installé dans le cloître de l'ancienne école pratique de Médecine (Université Paris VI). Reproduction: CLIN, Marie-Véronique. "De l'École de Chirurgie à l'École de Médecine». Universités et grandes écoles à Paris, Les palais de la Science. Paris : AAVP, 1999, p. 89-93, ill. p. 92.

49. Sur Farabeuf : HUGUET, Françoise. Les professeurs de la Faculté de Médecine de Paris, Dictionnaire biographique (1794-1939). Paris : INRP-CNRS, 1991, p. 181-182.

50. Reproduction : BALLOUL, Patrick. La salle de garde ou le plaisir des dieux. Urrugne : éd. de Loya, 1993, t. I, p. 86-87 et 90-91. L'ensemble de l'œuvre est signé « Sacha 90 ».

51. Voir : JOSSET, Patrice. La salle de garde, Histoire et signification des rituels des salles de garde de médecine, chirurgie et pharmacie, du Moyen-Âge à nos jours. Paris : Le Léopard d'Or, 1996, p. 50-60.

52. Voir : JOSSET, Patrice. La salle de garde, Histoire et signification des rituels des salles de garde de médecine, chirurgie et pharmacie, du Moyen-Âge à nos jours. Paris : Le Léopard d'Or, 1996, p. 52.

53. Témoignage oral, docteur J.C. B., 2000.

54. «Entretien avec le professeur Claude Degos ». Dans BALLOUL, Patrick. La salle de garde ou le plaisir des dieux. Édité par l'auteur, 1994, t. II, p. 103.

55. On peut se demander pourquoi Patrice Josset, qui livre une interprétation cohérente et complexe des rites des salles de garde, bien qu'elle mérite d'être discutée, ne traite quasiment pas des fresques et renvoie pour cette question au livre de Jacques Le Pesteur, lequel constitue pourtant une évocation plus qu'une tentative d'analyse et d'explication des fresques.

56. Une version remaniée et illustrée de ce texte a été publiée dans : «Fresques des salles de garde des hôpitaux parisiens - artistes et médecins, médecins artistes ». Labyrinthe, $\mathrm{n}^{\circ} 14$, hiver 2002-2003, p. 75-100. 


\section{RÉSUMÉS}

Discrètement licencieuses ou franchement pornographiques, les fresques des salles de garde des Internes en médecine intriguent, amusent, fascinent. De nombreux «beaux livres » et de savantes études ethnologiques leur ont été consacrés. L'approche retenue ici est autre: on a choisi de les considérer en tant qu'œuvres d'art, et, à ce titre, on s'est plus particulièrement intéressé au processus de commande par lequel un groupe d'internes choisit de travailler avec un artiste en vue de la réalisation d'une œuvre originale, destinée à marquer une étape de la vie du groupe. Chemin faisant, on découvrira les liens qui unissent artistes et médecins, qui s'expriment à travers les œuvres réalisées, mais aussi à travers les discours portés sur ces réalisations.

INDEX

Mots-clés : commande artistique, hôpital, Internat en médecine, sexualité

\section{AUTEUR}

\section{CHRISTIAN HOTTIN}

Conservateur du patrimoine, adjoint, département du pilotage de la recherche et de la politique scientifique, direction générale des patrimoines, ministère de la Culture et de la Communication christian.hottin@culture.gouv.fr 